\title{
Global optimization for multi-stage construction of rescue units in disaster response
}

\author{
Ning $\mathrm{Xu}^{\mathrm{a}}$, Qiong Zhang ${ }^{\mathrm{b}}$, Haoran Zhang ${ }^{\mathrm{c}, *}$, Minsung Hong ${ }^{\mathrm{d}}$, Rajendra Akerkar ${ }^{\mathrm{d}}$, Yongtu Liang ${ }^{\mathrm{a}}$ \\ ${ }^{a}$ Beijing Key Laboratory of Urban Oil and Gas Distribution Technology, China University of Petroleum-Beijing, Fuxue Road No. 18, Changping District, Beijing 102249, \\ China \\ ${ }^{\mathrm{b}}$ The University of Tokyo, 5-1-5 Kashiwanoha, Kashiwa, Chiba 277-8563, Japan \\ ${ }^{\mathrm{c}}$ Center for Spatial Information Science, The University of Tokyo, 5-1-5 Kashiwanoha, Kashiwa, Chiba 277-8563, Japan \\ ${ }^{\mathrm{d}}$ Western Norway Research Institute, P.O. Box 163, Sogndal 6851, Norway
}

\section{A R T I C L E I N F O}

\section{Keywords:}

Multi-stage construction

Global optimization

Bi-level decomposition algorithm

Accelerated solution

\begin{abstract}
A B S T R A C T
Disasters pose a serious threat to people' lives and urban environment, affecting the sustainable development of society. Then it's crucial to quickly develop an efficient rescue plan for the disaster area. However, disaster rescue is rather difficult due to the requirement to develop the optimal rescue plan as quickly as possible according to the information of trapped people and rescue teams, and the amount of information will continue to increase as the rescue proceeds. At present, most of the rescue plans are manually made based on previous rescue experience. But obviously these plans might be the not optimal one. Considering the real-time location data of trapped people, this paper develops a Mixed Integer Non-linear Programming (MINLP) model to find the highest efficient rescue plan To solve the model accurately and efficiently, a bi-level decomposition (BLD) algorithm is presented to iteratively solve a discretized Mixed Integer Linear Programming (MILP) model and its nonconvex Non-linear Programming (NLP) model until a converged solution is obtained. In addition, since more trapped people could be found over time, the built rescue units should also be considered when making a rescue plan for a new stage. To further improve the solving efficiency, an accelerated bi-level decomposition (ABLD) algorithm is also proposed. Finally, a real-world disaster rescue is given to validate the superiority of the proposed ABLD algorithm relative to particle swarm optimization (PSO) algorithm and BLD algorithm.
\end{abstract}

\section{Introduction}

Disasters usually cause a large number of casualties, economic losses or environmental pollution, affecting the sustainable development of the urban environment and society. Usually, disasters are able to be distinguished into natural disasters and man-made disasters. Natural disasters refer to earthquakes, floods, tsunamis, etc., whereas man-made disasters refer to explosions, fires, traffic disasters, and so on. To mitigate the consequences of the disaster, disaster operations management (DOM) has received extensive attention in OR/MS research (Altay \& Green, 2006; Galindo \& Batta, 2013). There are four phases included in DOM, namely mitigation, preparedness, response, and recovery. Mitigation refers to the reduction of natural or man-made risk factors (Okubo, 2016). To evaluate the program, policy environment, hazard mitigation, planning, emergency operation, reconstruction, as well as other themes will be considered (Chou, Hsu, Lin, Lee, \& Wei, 2017). Preparedness is to foster the ability to deal with various emergencies (Das, 2018). The response is to deploy resources timely to save lives and reduce losses when facing a disaster (Hayes \& Kelly, 2018). Recovery refers to both short-term behaviors and long-term activities (Jamali, Nejat, Ghosh, Jin, \& Cao, 2019; Miller, 2015). The former restore vital survival support system and the latter promote the restoration of normal social life.

This paper mainly focuses on the response phase because the most important thing is to implement rescue when a disaster strikes so that production and living activities can be restarted as soon as possible to ensure urban development. Through the above introduction, it can be seen that disasters have the characteristics of strong uncertainty and universality (Sun \& Li, 2014), resulting in the importance of efficient approaches to develop a rescue plan. In disaster management, decisionmaking is seen as a process evolving through problem definition, goal setting, project design, project selection, organization implementation, and feedback modification (Lei, Wu, Xu, \& Fujita, 2017). The process can be simplified into three stages according to the promotion of rescue

\footnotetext{
* Corresponding author.

E-mail address: zhang_ronan@csis.u-tokyo.ac.jp (H. Zhang).
} 


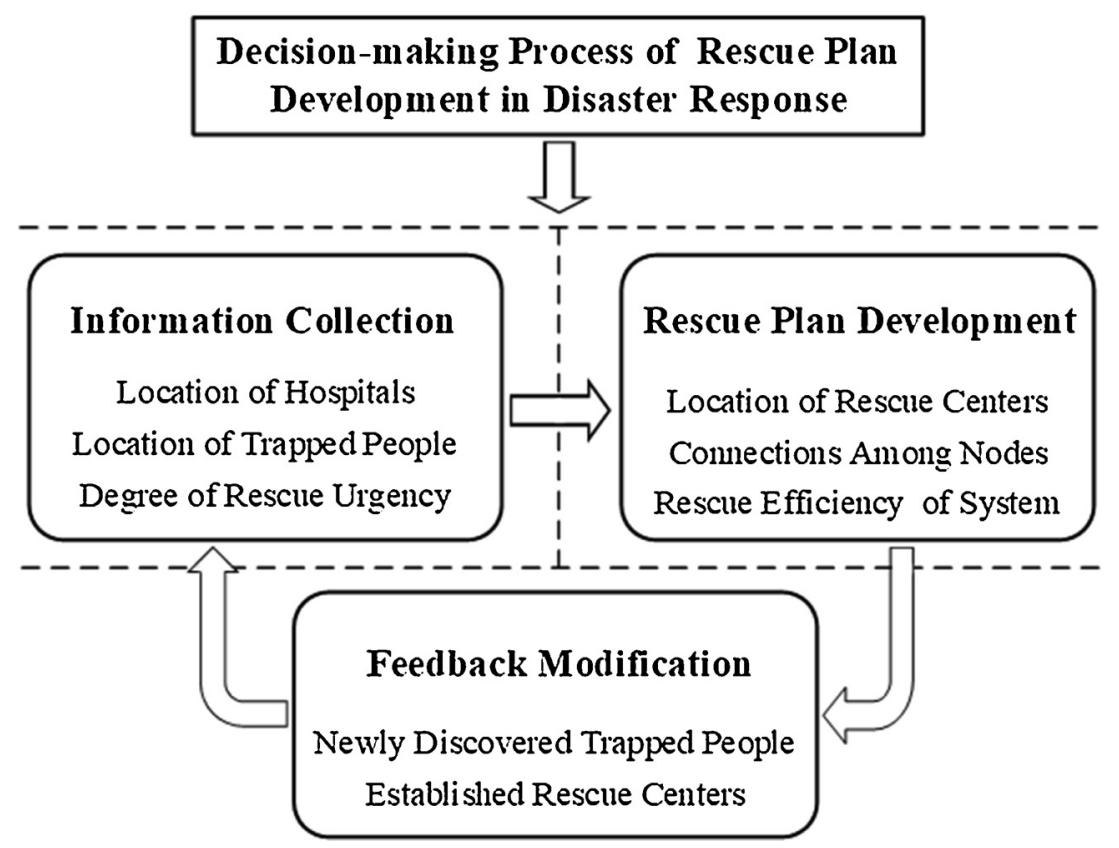

Fig. 1. Simplified decision-making process.

work, as shown in Fig. 1. The figure will be explained in detail in Section 3. For the rescue team, the priority is information collection which is the basis for implementing rescue. With the increasing adoption of new information and communication technologies (ICTs), such as social media and mobile devices, the role of regular citizens has become more influential in disaster response (Sommerfeldt, 2015; Zhang et al., 2019). In addition, it's also an important part of information that the integration of measures of physical, natural, and social systems by collecting and analyzing large-scale, heterogeneous, and high-resolution urban data (Kontokosta \& Malik, 2018). Based on the information collected, the next step is to develop a rescue plan with the determined goal to optimally allocate relief resources (Doan \& Shaw, 2019). Of course, the goal of the rescue plan can be single or multiple (Bozorgi-Amiri, Jabalameli, \& Al-E-Hashem, 2013). Considering that rescue will last for a period of time while the collected information keeps changing, it's necessary to modify the developed rescue plan according to the actual situation (Chen, Bai, \& Xing-Yuan, 2017). Only in this way can the rescue work be ensured to be high efficiently and constantly.

\section{Literature review}

In DOM, rescue units must be allocated efficiently to mitigate the consequences of disasters for cities and society. Generally, this issue involves multiple principals and multiple resources in the whole city, where a strong coupling relationship lies. For example, Wex, Schryen, Feuerriegel, and Neumann (2014) presented a problem which is a generalization of the parallel-machine scheduling problem, non-batch sequence-dependent setup times and a weighted sum of completion times. Therefore, it's usually difficult in terms of conceptualizing the problem or solving it.

When it comes to methodology, there are usually four main categories, including mathematical model, simulation, knowledge management, and group decision theory, of which mathematical model is the most widely used (Lei et al., 2017). Usually, rescue efficiency is taken as the goal of the established model when used to allocate the resources and for emergency and risk analysis (Wohlgemuth, 2012). Berkoune, Renaud, Rekik, and Ruiz (2012) proposed a model for a practical transportation problem by setting minimum response time as the objective. The problem is faced by rescue teams. Less response time means fewer losses in life and property. Moreover, the minimum social costs can also be taken as the objective (Afshar \& Haghani, 2012). For example, Pérez-Rodríguez and Holguín-Veras (2016) established an inventory-allocation-routing model to find the most cost-saving assignment of critical supplies. Of course, the objective of the problem can also be multiple (Abounacer, Rekik, \& Renaud, 2014). Tzeng et al. constructed a relief-distribution model for the design of relief delivery systems. The model has three objectives, namely the minimum total cost, minimum travel time and maximum satisfaction during the rescue plan (Tzeng, Cheng, \& Huang, 2007). In order to take more objectives into consideration, a Multi-Criteria Decision Making model (MCDM) that includes the value function concept is presented to reduce the negative impact of temporary housing (Amin Hosseini, de la Fuente, \& Pons, 2016). Besides, considering that the situation in the rescue work keeps changing, it's necessary to take the change into account in the model. There are two ways to consider the change, namely the uncertainty model and dynamic model. By setting the minimum total distance that vehicles traveled, Cavdur, Kose-Kucuk, and Sebatli (2016) developed a stochastic optimization model to allocate the temporary facilities in the disaster relief. To overcome the challenge of a more quantifiable analysis which cannot be captured by stochastic models, a statistical model was proposed to determine the location and distribution of relief supplies in DOM (Iqbal, Sardar, Lodhi, \& Hasan, 2018). A dynamic model was developed by Royero, Pacheco, and Pinedo (2016) to respond to the changing demand. To assess the evolution of resilience of drinking water supply system when disaster strikes, Alessandro Pagano et al. defined a System Dynamic Model and the model could adapt the infrastructure dynamically according to the change in the demand pattern (Pagano, Pluchinotta, Giordano, \& Vurro, 2017). In addition, the methodology to solve the problem based on simulation, knowledge management and group decision theory is very useful in some cases. For instance, Choi et al. (2018) presented a distributed simulation platform which could analyze facility damage comprehensively and respond to operational commands quickly.

No matter which way to conceptualize the problem, the established model is NP-hard (Wex et al., 2014), that is to say, it cannot be solved in polynomial time. Due to the urgency of disaster relief, the efficient solution of the model is necessary. The most common method in disaster relief is to propose a heuristic algorithm for the studied problem. $\mathrm{Hu}$ and Sheng (Hu \& Sheng, 2015) proposed a property model for the 
resource nodes by considering values of resources, disaster spread path and characteristics based on the principles of disaster spread. By this model, the optimal time needed in disaster relief and resource allocation can be obtained. Similarly, to develop a rescue plan during disaster rescue in maritime within a limited time, a heuristic project scheduling approach is proposed by Yan, Bao, Hu, and Ye (2009) which indicates high efficiency comparing to the manual formulation. Rauchecker and Schryen (2019) proposed a branch-and-price algorithm to get the schedule of rescue units after modeling the disaster situation as a binary linear minimization problem and the results could be obtained within ten minutes. To optimize the allocation of emergency rescue teams, Jian et al. (2018) adopted an auction-based task allocation scheme to obtain a cooperative rescue plan which performs better than F-MaxSum-based approach. In addition, instead of a heuristic algorithm, an intelligent algorithm is often adopted to solve the problem. When solving the multi-objective integer nonlinear programming model established, Zhang, Guo, Zhu, Yu, and Li (2017) proposed a method based on NSGA-II and C-METRIC whose performance was satisfactory in the solution. In the problem of generating exploratory trajectories for the networks which provide communication services, an algorithm based on PSO was developed by Sánchez-García, Reina, and Toral (2019) and the algorithm converges faster when comparing to an optimal trajectory planning algorithm.

Although the efficiency is greatly improved in solving the problem, the obtained solution is not optimal because it is not global optimization by adopting the heuristic algorithms and intelligent algorithms. In the problem discussed in this paper, the goal is to minimize the rescue time by determining the locations of rescue centers to allocation the rescue units in the disaster. This problem evolves from Capacitated Multi-facility Weber Problem (CMWP) which is NP-hard and the optimal solution cannot be obtained directly (Aras, Altınel, \& Orbay, 2007; Sherali, Al-Loughani, \& Subramanian, 2002). Here, BLD algorithm is adopted to solve the optimal solution after a certain number of iterations. And to improve efficiency, we propose an accelerated solution which performs better in model solving. Instead of adopting the uncertainty model and dynamic model, a multi-stage model is proposed in the paper to consider the change in a disaster situation. Each stage is based on the results of the previous stages. Mathematical models, including objective function and constraints, are established for the first stage and non-first stage respectively in Section 4.

The paper is organized as follows: In Section 3, we describe the studied problem and give the model requirements in detail. Section 4 establishes mathematical models for the first stage and non-first stage respectively, including objective function and constraints. In Section 5, the PSO algorithm and BLD algorithm are introduced to solve the mathematical model more efficiently, and meanwhile accelerated solutions are proposed as well. Section 6 presents a real case to evaluate the performance of the ABLD algorithm by comparing with PSO algorithm and BLD algorithm. Conclusions are provided in Section 7.

\section{Methodology}

\subsection{Problem description}

As mentioned above, to mitigate the consequences of disasters for cities and society, it's necessary to develop a rescue plan based on the known information collected and the resources available in the city for rescue. The efficiency of the rescue plan must be high considering that the less time it takes, the fewer losses in life and property. Besides, the model must be dynamic due to the constant change of the disaster situation. To meet the above requirements, a multi-stage model is established to develop the optimal rescue plan with an efficient algorithm. In particular, an MINLP model is established to determine the highest-efficient rescue plan, based on the relevant information of trapped people who have been found and the information of hospitals in the disaster area.

Relevant information of trapped people mainly includes two components, one is the current locations of the trapped and the other is the degree of urgency to rescue the trapped. The location information is useful for rescuers to find the trapped successfully while the degree of urgency determines the impact degree on the efficiency of the rescue plan. Relevant information of hospitals consists of two components as well, namely coordinates and the capacity to receive patients. The capacity is reflected by the number of beds it can provide.

Then, the development of rescue plan is divided into two parts. One is to determine the rescue centers' locations in the area, and the other is to determine the connections among hospitals, rescue centers and trapped people. The reason to set up the rescue center is that some trapped people are seriously injured and they need urgent treatment. It may take a long time to reach the nearest hospital. Therefore, they can be sent to the rescue centers for timely treatment firstly and then to the hospitals for more comprehensive treatment after the physical condition is stable. In conclusion, a high-efficient rescue plan should clearly illustrate that which rescue center or which hospital should trapped people be sent to.

Here, a simple schematic is taken to further explain the problem, as

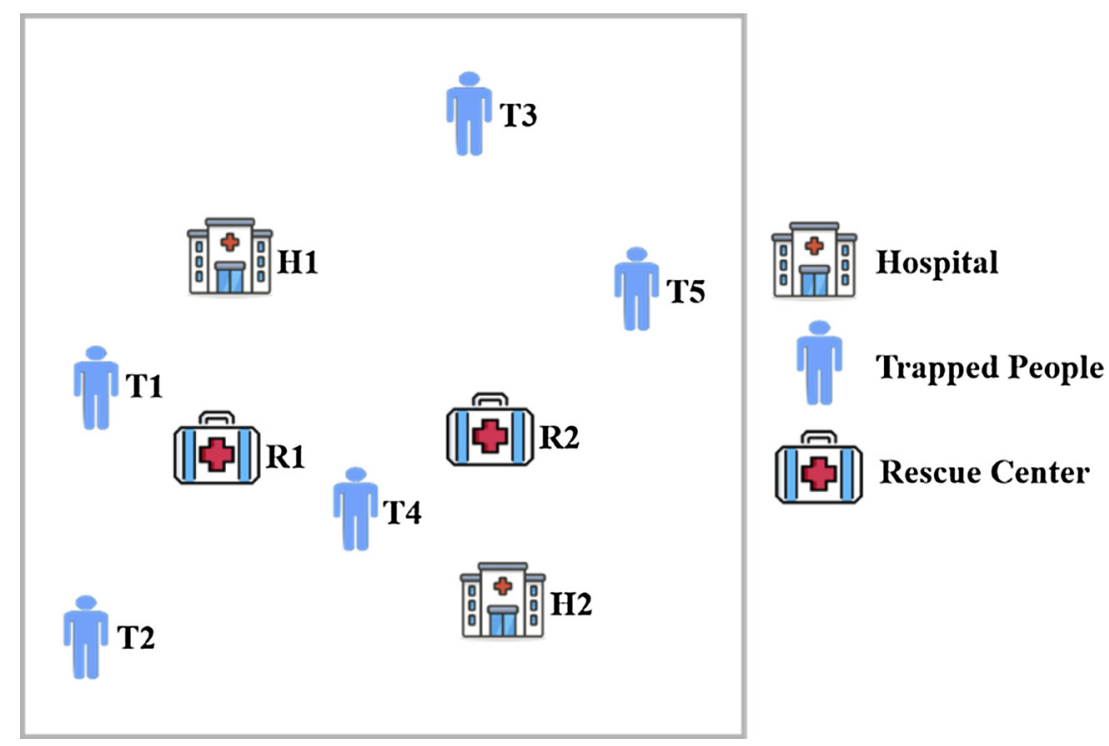

Fig. 2. Schematic diagram of the disaster area (1st stage). 
shown in Fig. 2. Firstly, the efficiency of the rescue plan depends on the traveling distance from the places of trapped people to hospitals. The shorter distance it takes, the higher the efficiency it has. The hospitals' ( $\mathrm{H} 1$ and H2) coordinates and a number of beds, as well as trapped people's (T1, T2, et al.) locations and degree of urgency, are known in the disaster area. Then, the rescue plan is developed by the established mathematical model. Rescue centers (R1 and R2) act as the hubs to connect hospitals and trapped people, and their locations have a significant impact on the efficiency of the rescue plan. Beyond that, the rescue efficiency is also affected by the rescue scheme, i.e. the assignment of rescue centers and hospitals to all trapped people. To be specific, each trapped person should be specified to one rescue center or one hospital, and all the people at one rescue center should be sent to the same hospital. For example, T2 is connected to R1 while R1 is connected to H1, indicating that T2 is sent to rescue center R1 first and to $\mathrm{H} 1$ afterward. Generally, one rescue center will not be set up if it does not provide rescue services to any trapped person. Moreover, a trapped person nearby a hospital can be sent to the hospital without going through any rescue center directly. The objective function of the studied issue is to work out the most efficient rescue plan that meets all the above requirements.

Usually, more trapped people will be found as the rescue proceeds. In response to this dynamic process, the rescue plan can be divided into multi-stages. As mentioned above, some rescue centers are set up and the connections among hospitals, rescue centers and trapped people are determined in the first stage in order to save the discovered people. When developing a rescue plan for the non-first stage, the only difference is that all the rescue centers which have been set up before should be taken into consideration in the mathematical model. Just as shown in Fig. 3, rescue center R1 has been set up in the previous stage and it should be a part of the non-first stage rescue plan development. In the process, eight trapped people (T1-T8) and two possible locations of rescue centers (R2 and R3) need to be considered. Due to the existence of R1, T5 can be connected to R1 instead of being connected to R3 or $\mathrm{H} 1$ directly. In this way, the efficiency of the non-first stage is improved relative to not considering the rescue centers which have been set up. At the same time, the problem becomes more complicated.

\subsection{Model requirements}

(A) Assumptions:

- The distance between two locations is expressed as a Euclidean distance in the two-dimensional disaster area.

- The speed of rescue vehicles is constant in different regions of the disaster area.

- Since only emergency services are provided, there is no upper limit on the number of trapped people admitted to one rescue center.

- The vehicles are adequate to meet the demand.

\section{(B) Given:}

- Coordinates of hospitals in the disaster area, which are represented by two arrays of $\mathrm{x}$ and $\mathrm{y}$.

- The capacity of providing service for the trapped people of hospitals in the disaster area, reflected by the number of beds they own.

- Coordinates of trapped people to be rescued, which are represented by two arrays of $\mathrm{x}$ and $\mathrm{y}$.

- Number of trapped people found at the different locations in the disaster area.

- Degree of urgency to rescue the trapped people found at the different locations in the disaster area.

- The time needed to set up a rescue center and the speed of rescue vehicles.

- Coordinates of rescue centers which have been set up when developing the non-first stage rescue plan.

\section{(C) Determine:}

- Locations of the rescue centers in the disaster area.

- Optimal rescue plan with the highest efficiency, reflected in the connections among hospitals, rescue centers and trapped people.

\section{Mathematical model}

In the rescue plan development problem, the highest efficiency of the rescue plan is set as the object. And the model established is to find the connections among hospitals, rescue centers and trapped people under the condition that the capacity of facilities will not be exceeded. For example, the number of trapped people sent to the hospital cannot exceed its capacity. The objective function and constraints are shown below.

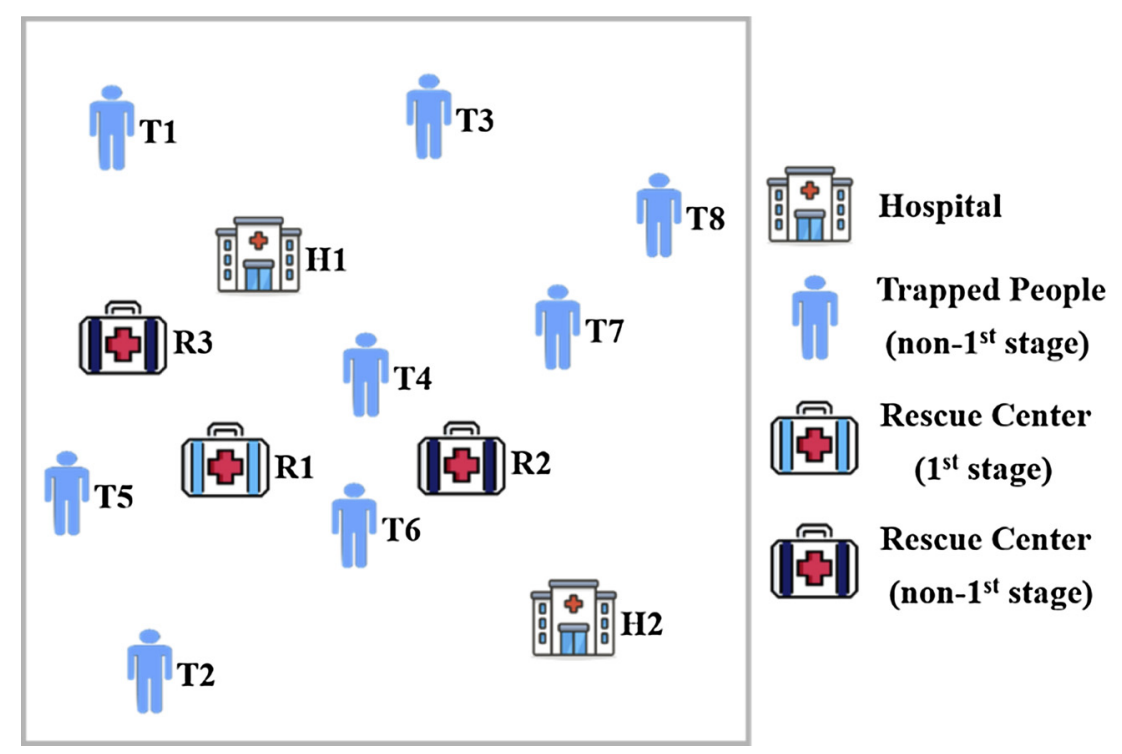

Fig. 3. Schematic diagram of the disaster area (non-1st stage). 


\subsection{The first stage}

\subsubsection{Objective function}

As mentioned above, the efficiency of the rescue plan is reflected by how much distance it takes to rescue the trapped people and the less distance it takes, the higher efficiency it has. Then, the total distance taken in the rescue plan is composed of four parts, just as shown in constraint (1a). The detailed explanation is shown by constraints (1b)-(1e).

$\min f=\sum_{k \in K} f 1_{k}+\sum_{i \in I} \sum_{j \in J} f 2_{i, j}+\sum_{k \in K} \sum_{j \in J} f 3_{k, j}+\sum_{i \in I} \sum_{k \in K} f 4_{i, k}$

\subsubsection{Constraints}

It takes some time to set up the rescue centers. And the time can be different when setting up rescue centers in different regions. $P_{k}^{U}$ represents the time needed to set up a rescue center at a possible location $k$ and $V$ represents the speed of rescue vehicles. Considering that the efficiency of the rescue plan is represented by distance, it's necessary to express the time with an equivalent distance by the speed of rescue vehicles. And it's shown in constraint (1b). If a rescue center is set up at a possible location $k, B_{k}^{W}$ equals 1 .

$f 1_{k}=V P_{k}^{U} B_{k}^{W} \quad k \in K$

Constraint (1c) indicates that it can be determined that the distance taken to transport the injured from trapped locations to a hospital. $D_{i, j}$ represents the distance between the hospital $i$ and trapped person $j$. If the trapped person $j$ is taken to the hospital $i, B_{i, j}^{S}$ equals 1 . Especially, taking into account the difference in the degree of injury, degree of urgency is considered in the model, which is represented by $\alpha_{j}$. And the larger the value of $\alpha_{j}$ is, the more urgent it is to rescue the trapped person $j$.

$f 2_{i, j}=\alpha_{j} B_{i, j}^{S} D_{i, j} \quad i \in I, \quad j \in J$

When the trapped people are too far from the hospital, a rescue center will be set up to improve the efficiency of the rescue system. Trapped people will be transported to a rescue center for emergency treatment before being sent to a hospital. Just as constraint (1c), it can be determined that the distance is taken to arrive at a rescue center and hospital in constraints (1d) and (1e) respectively. The meanings of components are similar to those in constraint (1c).

$f 3_{k, j}=\alpha_{j} B_{k, j}^{S N} D_{k, j} \quad k \in K, j \in J$

$f 4_{i, k}=N_{i, k}^{C} D_{i, k} \quad i \in I, \quad k \in K$

$B_{k}^{W}$ is a binary variable indicating whether to set up a rescue center at a possible location $k$ or not. Constraint (1f) indicates that if there is not a rescue center set up at possible location $k$, the rescue center will not transport the injured to any hospital. On the contrary, if a rescue center is set up at a possible location $k$, the rescue center will transport the injured to only one hospital while one hospital could receive patients from several rescue centers.

$\sum_{i \in I} B_{i, k}^{S M}=B_{k}^{W} \quad k \in K$

Constraint $(1 \mathrm{~g})$ indicates that only when a rescue center is set up at a possible location $k$ can it provide rescue services for the tapped people nearby. From the constraint, it can be seen that there is no upper limit on the capacity of the rescue center because the constraints on traffic resources are not considered in the model. Therefore, the people can be transferred to hospital from rescue center at any time or simultaneously as long as there are enough vehicles. And each trapped person must be rescued by one center or one hospital which is constrained in constraint (1h). $M$ represents the sufficiently large number. $\sum_{j \in J} B_{k, j}^{S N} \leq B_{k}^{W} M \quad k \in K$

$\sum_{i \in I} B_{i, j}^{S}+\sum_{k \in K} B_{k, j}^{S N}=1 \quad j \in J$

Considering that there is a limited number of the beds available for injured people in one hospital, represented by $C_{i}^{A}$, the number of trapped people sent to the hospital should be less than the maximum capacity, as shown in constraint (1i). $P_{j}^{E}$ represents the number of trapped people found at the location $j$. And constraints $(1 \mathrm{j})$ and $(1 \mathrm{k})$ determine if an injured person $j$ is taken to hospital $i$ via rescue center $k$ or not.

$\sum_{k \in K} \sum_{j \in J} P_{j}^{E} B_{i, k, j}^{S M N}+\sum_{j \in J} P_{j}^{E} B_{i, j}^{S} \leq C_{i}^{A} \quad i \in I$

$B_{i, k, j}^{S M N} \leq \frac{B_{i, k}^{S M}+B_{k, j}^{S N}}{2} \quad i \in I, \quad k \in K, j \in J$

$B_{i, k, j}^{S M N} \geq \frac{B_{i, k}^{S M}+B_{k, j}^{S N}-1}{2} \quad i \in I, \quad k \in K, \quad j \in J$

The distance between hospitals and rescue centers is determined in constraint (11), while that between rescue centers and injured people is determined by constraint $(1 \mathrm{~m}) . x_{k}$ and $y_{k}$ represents the coordinates of possible location of the rescue center $k$.

$D_{i, k}=\sqrt{\left(x_{i}-x_{k}\right)^{2}+\left(y_{i}-y_{k}\right)^{2}} \quad i \in I, \quad k \in K$

$D_{k, j}=\sqrt{\left(x_{k}-x_{j}\right)^{2}+\left(y_{k}-y_{j}\right)^{2}} \quad k \in K, \quad j \in J$

\subsection{Non-first stage}

As the rescue proceeds, more trapped person will be found, which means more rescue centers need to be set up. When planning layout of new rescue centers for the non-first stage, the built centers have an impact on the plan and need to be considered in the mathematical model, as expressed in (2a)-(2s).

Comparing with Section 4.1, there are some points worth noting when establishing the model for the non-first stage because of the rescue centers set up in the previous stages. In the model, $K 1$ represents the set of the locations where rescue centers have been set up in the previous stage and $K 2$ represents the set of the locations where rescue centers are set up in the non-first stage. Only the coordinates of the rescue centers set up in the current stage need to be determined. Relatively, the coordinates of the rescue centers set up in the previous stage are known. Based on the above facts, the time needed to set up the rescue centers can be saved since parts of them have been set up already in the previous stages. Then, the time to be considered is just about that needed to set up the possible rescue centers which are shown in constraint (2b). Similarly, due to the known coordinates of the rescue centers, the distance between them and other nodes can be obtained directly, just as shown in constraints (2d) and (2f). When it comes to the connection, only when there are trapped people sent to the rescue center can it be connected to one hospital. The relationship is represented by constraints (2h) and (2i) and they contrast with constraints $(2 \mathrm{j})$ and $(2 \mathrm{k})$.

$$
\begin{aligned}
\min f= & \sum_{k^{\prime}=k 1_{\max }+1}^{k 2_{\max }} f 1_{k^{\prime}}+\sum_{i \in I} \sum_{j \in J} f 2_{i, j} \\
& +\sum_{k 1 \in K 1} \sum_{j \in J} f 3_{k 1, j}+\sum_{k^{\prime}=k 1_{\max }+1}^{k 2_{\max }} \sum_{j \in J} f 3_{k^{\prime}, j}+\sum_{i \in I} \sum_{k 1 \in K 1} f 4_{i, k 1} \\
& +\sum_{i \in I} \sum_{k^{\prime}=k 1_{\max }+1}^{k 2_{\max }} f 4_{i, k^{\prime}} \\
f 1_{k^{\prime}}= & V P_{k^{\prime}}^{U} B_{k^{\prime}}^{W} \quad k 1_{\max }<k^{\prime} \leq k 2_{\max }
\end{aligned}
$$




$$
\begin{aligned}
& f 2_{i, j}=\alpha_{j} B_{i, j}^{S} D_{i, j} \quad i \in I, j \in J \\
& f 3_{k 1, j}=\alpha_{j} B_{k 1, j}^{S N} D_{k 1, j} \quad k 1 \in K 1, j \in J \\
& f 3_{k^{\prime}, j}=\alpha_{j} B_{k^{\prime}, j}^{S N} D_{k^{\prime}, j} \quad k 1_{\max }<k^{\prime} \leq k 2_{\max }, \quad j \in J \\
& f 4_{i, k 1}=B_{i, k 1}^{S M} D_{i, k 1} \quad i \in I, \quad k 1 \in K 1 \\
& f 4_{i, k^{\prime}}=B_{i, k^{\prime}}^{S M} D_{i, k^{\prime}} \quad i \in I, k 1_{\max }<k^{\prime} \leq k 2_{\max } \\
& \sum_{i \in I} B_{i, k 1}^{S M} \leq 1 \quad k 1 \in K 1
\end{aligned}
$$$$
\sum_{i \in I} B_{i, k 1}^{S M} M \geq \sum_{j \in J} B_{k 1, j}^{S N} \quad k 1 \in K 1
$$$$
\sum_{i \in I} B_{i, k^{\prime}}^{S M}=B_{k^{\prime}}^{W} \quad k 1_{\max }<k^{\prime} \leq k 2_{\max }
$$$$
\sum_{j \in J} B_{k^{\prime}, j}^{S N} \leq B_{k^{\prime}}^{W} M \quad k 1_{\max }<k^{\prime} \leq k 2_{\max }
$$$$
\sum_{i \in I} B_{i, j}^{S}+\sum_{k 2 \in K 2} B_{k 2, j}^{S N}=1 \quad j \in J
$$$$
\sum_{k 2 \in K 2} \sum_{j \in J} P_{j}^{E} B_{i, k 2, j}^{S M N}+\sum_{j \in J} P_{j}^{E} B_{i, j}^{S} \leq C_{i}^{A} \quad i \in I
$$$$
B_{i, k 2, j}^{S M N} \leq \frac{B_{i, k 2}^{S M}+B_{k 2, j}^{S N}}{2} \quad i \in I, \quad k 2 \in K 2, j \in J
$$$$
B_{i, k 2, j}^{S M N} \geq \frac{B_{i, k 2}^{S M}+B_{k 2, j}^{S N}-1}{2} \quad i \in I, \quad k 2 \in K 2, j \in J
$$$$
D_{i, k^{\prime}}=\sqrt{\left(x_{i}-x_{k^{\prime}}\right)^{2}+\left(y_{i}-y_{k^{\prime}}\right)^{2}} \quad i \in I, \quad k 1_{\max }<k^{\prime} \leq k 2_{\max },
$$$$
D_{k^{\prime}, j}=\sqrt{\left(x_{k^{\prime}}-x_{j}\right)^{2}+\left(y_{k^{\prime}}-y_{j}\right)^{2}} \quad k 1_{\max }<k^{\prime} \leq k 2_{\max }, j \in J
$$

\section{Model solving}

In the objective function mentioned in Section 4.1.1, $B_{i, k}^{S M}$ and $D_{i, k}$ are both variables which should be determined in the MINLP model at the same time. However, as we known, it's difficult to solve a MINLP model, reflected in that it's usually difficult to find a suitable commercial solver for the model with nonlinearity and it often takes a long time to solve the model with large scale. Therefore, converting the established MINLP model into MILP model is necessary in the problem. Two methods are adopted, as shown below.

\subsection{PSO algorithm}

In PSO algorithm, $D_{i, k}$ can be obtained in advance by determining the coordinates of the rescue centers so that the MINLP model can be converted to an MILP model. Fig. 4 shows the flow chart. The process of the rescue system design can be divided into two steps as well. Firstly, possible locations of rescue centers can be determined by PSO algorithm, which can generate the coordinates by corresponding strategy. Secondly, based on the locations of the possible rescue centers, an MILP model will be naturally obtained which sets the rescue efficiency of system as the objective function and takes the existing locations of hospitals and other requirements into consideration. Then, in each iteration, information of particles will be updated according to the optimal value of the MILP model which is set as the fitness of PSO algorithm. When the convergence requirements of PSO algorithm can be met, the optimal rescue plan, including locations of rescue centers and connection among trapped people, rescue centers and hospitals, will be obtained finally.

\subsection{Bi-level decomposition algorithm}

BLD algorithm is proposed by Lara, Trespalacios, and Grossmann (2018) to solve the Capacitated Multi-facility Weber Problem (CMWP). The problem is to determine locations in continuous 2-dimensional space for opening new facilities that are connected to supply and customer nodes. Inspired by this, the primary MINLP model established in Section 4 can be decomposed into a discretized MILP model and its nonconvex NLP model which are shown below.

Similar to the PSO algorithm, the MINLP model can be converted into an MILP model by determining $B_{i, k}^{S M}$ in advance using BLD algorithm. In the BLD algorithm, when a disaster area is divided into multiple blocks, just as shown in Fig. 5, the problem of where to set up a rescue center is transformed into that of which block to be selected to set up a rescue center. $B_{i, k}^{S M}, B_{k, j}^{S N}$ and $B_{i, j}^{S}$ can be obtained in advance by solving the MILP model. Based on the results, the detailed locations of rescue centers can be determined by solving the nonconvex NLP model. Table 1 shows the process. According to the proof (Lara et al., 2018), a lower bound to the MINLP problem is yielded by the MILP model and an upper bound will be yielded by the NLP model. As the number of blocks divided in the area increases, the upper and lower bound will get closer. When the difference between the lower and upper bound, which is represented by gap, reaches the pre-specified optimality tolerance, BLD algorithm can be seen converged and then the optimal results can be obtained.

\subsubsection{MILP model}

When the area has been divided into multiple blocks, the position of each block is fixed and it means that the range of distance among hospitals, trapped people and blocks can be determined. By adopting the shortest distance, $D_{i, r}^{L}$ and $D_{j, r}^{L}$ in constraints (3d) and (3e), the MILP model can be developed, which is expressed in (3a)-(3k). The shortest distance means the minimum Euclidean distance between blocks and hospitals or trapped people, for example, the red line AB in Fig. 4 means the shortest distance between $\mathrm{H} 1$ and the block where T3 located. For that reason, the optimal solution of MILP model will yield a lower bound to the primary MINLP model. In addition, considering that a rescue center can only be set up at one block, the other three constraints (31)-(3n) should be added to the MILP model. Considering that when the model scale of MILP is large, it will take very long time to solve the model completely. To avoid this, the maximum time to solve the MILP model in each iteration is set to be less than $150 \mathrm{~s}$.

$$
\begin{aligned}
& \min f=\sum_{k \in K} \sum_{r \in R} f 1_{k, r}+\sum_{i \in I} \sum_{j \in J} f 2_{i, j} \\
& +\sum_{k \in K} \sum_{j \in J} \sum_{r \in R} f 3_{k, j, r}+\sum_{i \in I} \sum_{k \in K} \sum_{r \in R} f 4_{i, k, r} \\
& f 1_{k, r}=V P_{U} B_{W} \quad k \in K, \quad r \in R \\
& f 2_{i, j}=\alpha_{j} B_{i, j}^{S} D_{i, j} \quad i \in I, j \in J \\
& f 3_{k, j, r}=\alpha_{j} B_{k, j, r}^{S N} D_{j, r}^{L} \quad k \in K, \quad j \in J, \quad r \in R \\
& f 4_{i, k, r}=B_{i, k, r}^{S M} D_{i, r}^{L} \quad i \in I, \quad k \in K, \quad r \in R \\
& \sum_{i \in I} B_{i, k, r}^{S M}=B_{k, r}^{W} \quad k \in K, \quad r \in R \\
& \sum_{j \in J} B_{k, j, r}^{S N} \leq B_{k, r}^{W} M \quad k \in K, \quad r \in R \\
& \sum_{i \in I} B_{i, j}^{S}+\sum_{k \in K} \sum_{r \in R} B_{k, j, r}^{S N}=1 \quad j \in J \\
& \sum_{k \in K} \sum_{j \in J} P_{j}^{E} B_{i, k, j}^{S M N}+\sum_{j \in J} P_{j}^{E} B_{i, j}^{S} \leq C_{i}^{A} \quad i \in I
\end{aligned}
$$




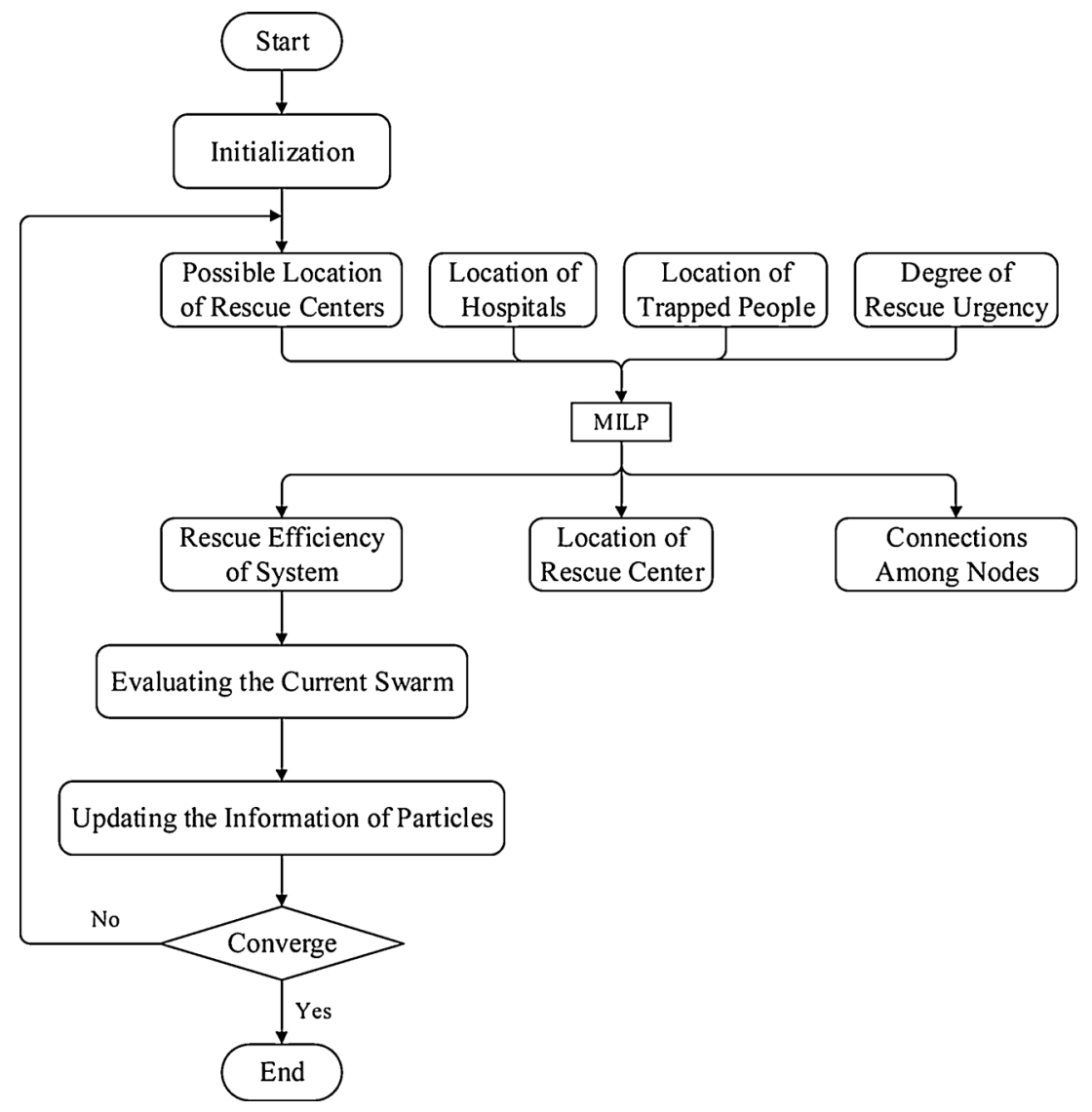

Fig. 4. Flow chart of the design by PSO algorithm.

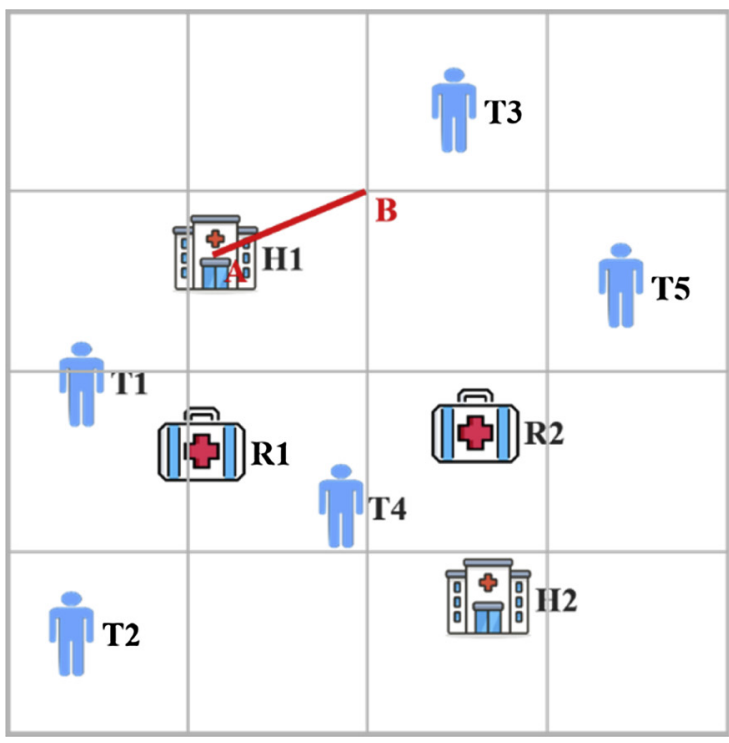

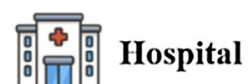

Trapped People

||⿴囗十) Rescue Center

Fig. 5. Schematic diagram of the disaster area after the division (1st stage).

$B_{i, k, j}^{S M N} \leq \frac{\sum_{r \in R} B_{i, k, r}^{S M}+\sum_{r \in R} B_{k, j, r}^{S N}}{2} \quad i \in I, \quad k \in K, \quad j \in J$

$B_{i, k, j}^{S M N} \geq \frac{\sum_{r \in R} B_{i, k, r}^{S M}+\sum_{r \in R} B_{k, j, r}^{S N}-1}{2} \quad i \in I, \quad k \in K, \quad j \in J$

$\sum_{r \in R} B_{k, r}^{W} \leq 1 \quad k \in K$

$$
\begin{aligned}
& \sum_{r \in R} B_{i, k, r}^{S M} \leq 1 \quad i \in I, \quad k \in K \\
& \sum_{r \in R} B_{k, j, r}^{S N} \leq 1 \quad k \in K, \quad j \in J
\end{aligned}
$$

\subsubsection{NLP model}

By solving the MILP model, it can be determined which block the rescue center should be set up at. The next task is to determine the 
Table 1

Procedure of rescue plan development in BLD algorithm.

Algorithm Global optimization for multi-stage construction of rescue centers in the event of disaster

1: Set iteration iter $=1$, gap $=100 \%$, and a pre-specified optimality tolerance $\varepsilon$;

2: Divide the disaster area into multiple blocks in $m \times n$

3: while gap $>\varepsilon$ do

4: Solve the MILP model and yield a Lower Bound $(L B)$

5: Fix the decision of which facilities $k$ to build;

6: Fix the decisions of how to allocate the rescue mission $B_{i, k}^{S M}$ and $B_{k, j}^{S N}$.

7: $\quad$ Solve the NLP subproblem and compute an Upper Bound (UB);

8: $\quad$ gap $=(U B-L B) / U B$;

9: $\quad m=m+N 1$ and $n=n+N 2$, where $N 1, N 2 \in N$

10: end while

detailed location of the rescue center in the block. Based on the known information, the NLP model is established which is expressed in (4a) $-(4 g)$. Due to the existence of equations (4d) and (4e), the model is non-linear and non-convex because there are multiple extreme points in the feasible region. In addition, the solution of the NLP model will be an upper bound to the primary MINLP model which means it will be greater than or equal to that of MINLP model because the model may not be solved completely. Considering which block a rescue center will be set up is determined, the coordinates of each rescue center are limited to a range, just as shown is constraints (4f) and (4g).

$$
\begin{aligned}
& \min f=\sum_{k \in K} f 1_{k}+\sum_{i \in I} \sum_{j \in J} f 2_{i, j}+\sum_{i \in I} \sum_{k \in K} f 2_{i, k}+\sum_{k \in K} \sum_{j \in J} f 3_{k, j} \\
& f 1_{k}=V P_{k}^{U} B_{k}^{W} \quad k \in K \\
& f 2_{i, j}=\alpha_{j} B_{i, j}^{S} D_{i, j} \quad i \in I, j \in J \\
& f 3_{k, j}=\alpha_{j} B_{k, j}^{S N} D_{k, j} \quad k \in K, j \in J \\
& f 4_{i, k}=B_{i, k}^{S M} D_{i, k} \quad i \in I, \quad k \in K \\
& D_{i, k}=\sqrt{\left(x_{i}-x_{k}\right)^{2}+\left(y_{i}-y_{k}\right)^{2}} \quad i \in I, k \in K \\
& D_{k, j}=\sqrt{\left(x_{k}-x_{j}\right)^{2}+\left(y_{k}-y_{j}\right)^{2}} \quad k \in K, j \in J \\
& x_{\text {down }} \leq x_{k} \leq x_{\text {up }} \quad k \in K \\
& y_{\text {down }} \leq y_{k} \leq y_{\text {up }} \quad k \in K
\end{aligned}
$$

\subsection{Accelerated solution}

Considering that a reasonable rescue plan based on BLD algorithm is necessary for the rescue team to carry out rescue work, an efficient and accurate method to solve the MINLP model established in Section 4 is important. Although BLD algorithm is a great way to solve the model, there are two serious problems in efficiency, one is that the scale of the model becomes larger and larger as the number of blocks increase, and the other is difficulty in convergence. To solve the problems, two measures are adopted to reduce the model scale in the process of solving and speed up the convergence. Then, the ABLD algorithm appears.

\subsubsection{Service scope}

To improve the rescue efficiency of plan, trapped people will be usually sent to the nearby hospital since there are many hospitals in the disaster area. Therefore, connection will not appear between two nodes that are too far from each other. In this problem, we take it as the service scope of the hospitals. In other words, the service scope of hospital $i$ via rescue center $\mathrm{k}$ means the area where rescue centers can be connected to it, for example, area S1 represents the service scope of H1 in Fig. 6. Similarly, the service scope of rescue center k means the area where trapped people can be connected to it, just as area S2 in Fig. 6 which represents the service scope of R2.

Based on the above statement, set $R C$ and $R P$ are introduced to the model expressed by (5a)-(5n), which represent the blocks that could be served by each hospital and each rescue center respectively. In addition, hospitals of different sizes have different service scope, reflected in that the more beds one hospital owns, the larger its service scope is. Due to the introduction of the two sets, the model scale is reduced greatly and the efficiency of solving is obviously improved.

$$
\begin{aligned}
& \min f= \sum_{k \in K} \sum_{r \in R} f 1_{k, r}+\sum_{i \in I} \sum_{j \in J} f 2_{i, j} \\
&+\sum_{k \in K} \sum_{j \in J} \sum_{r c \in R C} f 3_{k, j, r c}+\sum_{i \in I} \sum_{k \in K} \sum_{r p \in R P} f 4_{i, k, r p} \\
& f 1_{k, r}=V P_{U} B_{W} \quad k \in K, \quad r \in R \\
& f 2_{i, j}=\alpha_{j} B_{i, j}^{S} D_{i, j} \quad i \in I, \quad j \in J \\
& f 3_{k, j, r c}=\alpha_{j} B_{k, j, r c}^{S N} D_{j, r c}^{L} \quad k \in K, \quad j \in J, \quad r c \in R C \\
& f 4_{i, k, r p}=B_{i, k, r p}^{S M} D_{i, r p}^{L} \quad i \in I, \quad k \in K, \quad r p \in R P \\
& \sum_{i \in I} B_{i, k, r p}^{S M}=B_{k, r p}^{W} \quad k \in K, \quad r p \in R P
\end{aligned}
$$

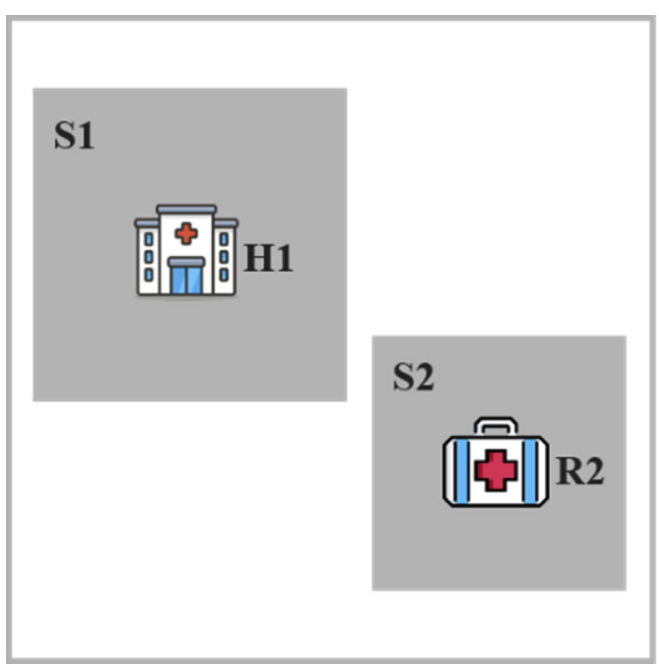

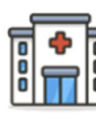

\section{Hospital}

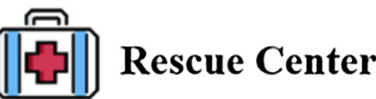

Fig. 6. Service scope. 


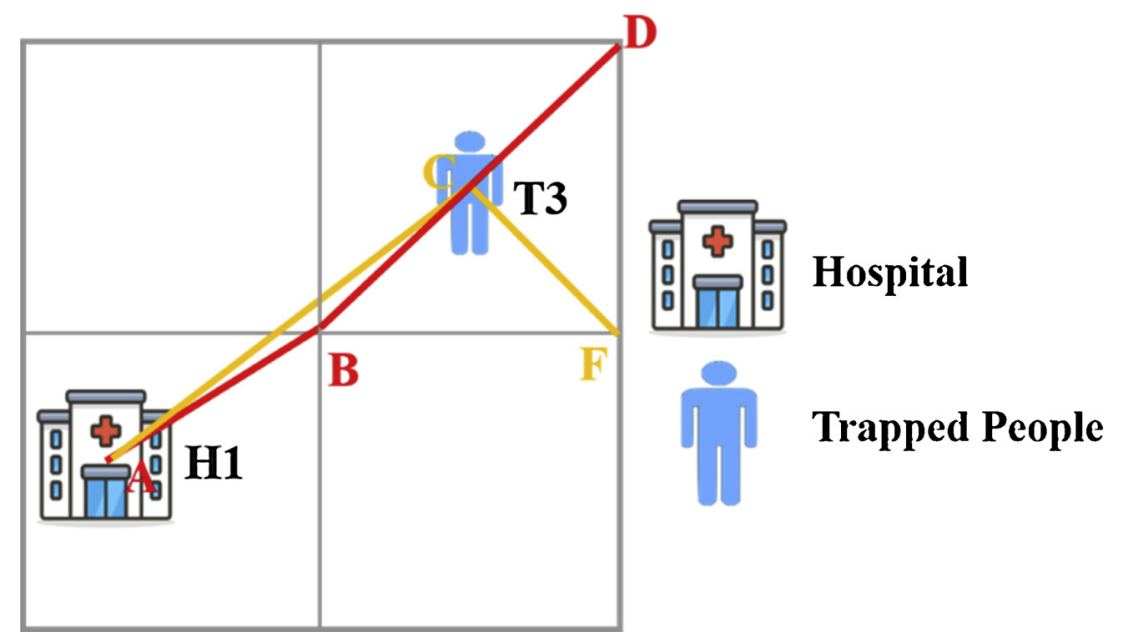

Fig. 7. Median distance.

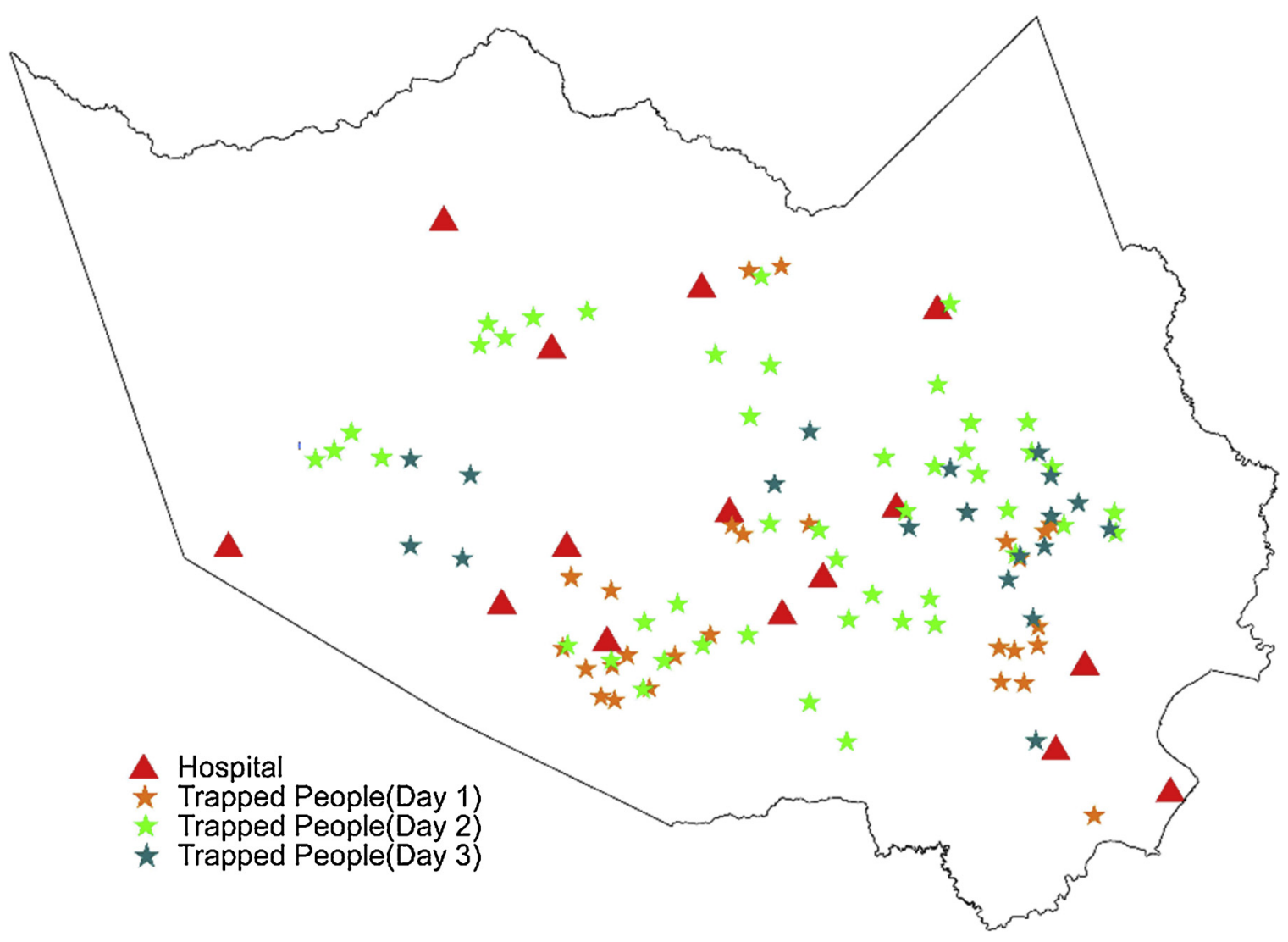

Fig. 8. Schematic diagram for the layout of the system.

Table 2

Information of hospitals.

\begin{tabular}{llllllll}
\hline Hospital & $\mathrm{X} / \mathrm{m}$ & $\mathrm{Y} / \mathrm{m}$ & Beds & Hospital & $\mathrm{X} / \mathrm{m}$ & $\mathrm{Y} / \mathrm{m}$ & Beds \\
\hline H1 & 37,936 & 20,669 & 3926 & H9 & 0 & 27,631 & 208 \\
H2 & 40,734 & 24,478 & 715 & H10 & 58,675 & 15,396 & 400 \\
H3 & 32,415 & 54,421 & 430 & H11 & 14,751 & 61,312 & 358 \\
H4 & 18,721 & 21,733 & 302 & H12 & 64,532 & 2309 & 531 \\
H5 & 34,317 & 31,224 & 260 & H13 & 22,136 & 48,128 & 313 \\
H6 & 23,165 & 27,641 & 426 & H14 & 45,757 & 31,712 & 250 \\
H7 & 56,674 & 6617 & 293 & H15 & 25,932 & 17,885 & 547 \\
H8 & 48,564 & 52,159 & 255 & & & & \\
\hline
\end{tabular}

Table 3

Parameters of the established model.

\begin{tabular}{|c|c|c|c|c|c|}
\hline $\begin{array}{l}\text { Item } \\
\text { Value }\end{array}$ & $\begin{array}{l}\mathrm{V}(\mathrm{km} / \mathrm{h}) \\
60\end{array}$ & $\begin{array}{l}\mathrm{P}^{\mathrm{U}}(\min ) \\
30\end{array}$ & $\begin{array}{l}\varepsilon \\
10 \%\end{array}$ & $\begin{array}{l}\mathrm{S}^{\mathrm{H}}(\mathrm{km}) \\
20\end{array}$ & $\begin{array}{l}\mathrm{S}^{\mathrm{RC}}(\mathrm{km}) \\
15\end{array}$ \\
\hline & ${ }_{r}^{W} r c$ & $\quad r c \in I$ & & & $(5 g$ \\
\hline$\sum_{i \in I} B_{i, j}^{S}$ & $\sum_{K} B_{k}$ & $=1 j \in$ & & & $(5 \mathrm{~h}$ \\
\hline$\sum_{k \in K} \sum_{j \in .}$ & ${ }_{k, j}^{M N}+\sum_{j}$ & $B_{i, j}^{S} \leq C_{i}^{A}$ & & & (5i \\
\hline
\end{tabular}




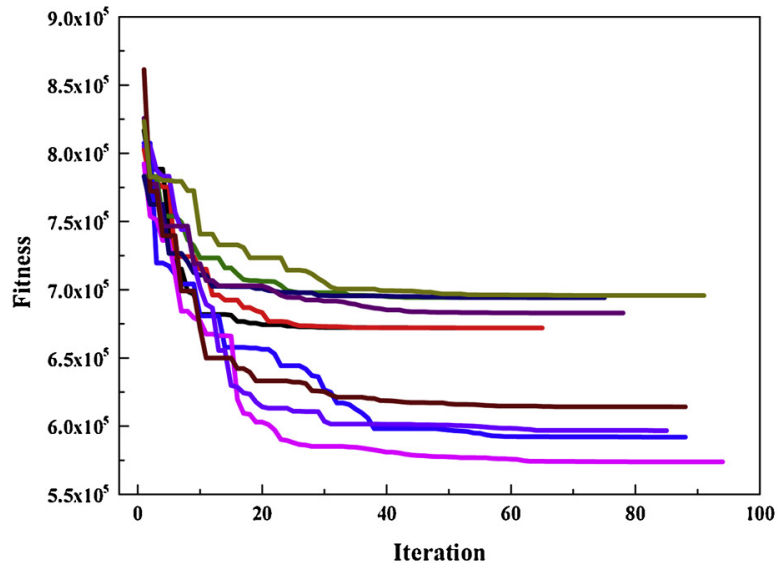

( a ) GPSO

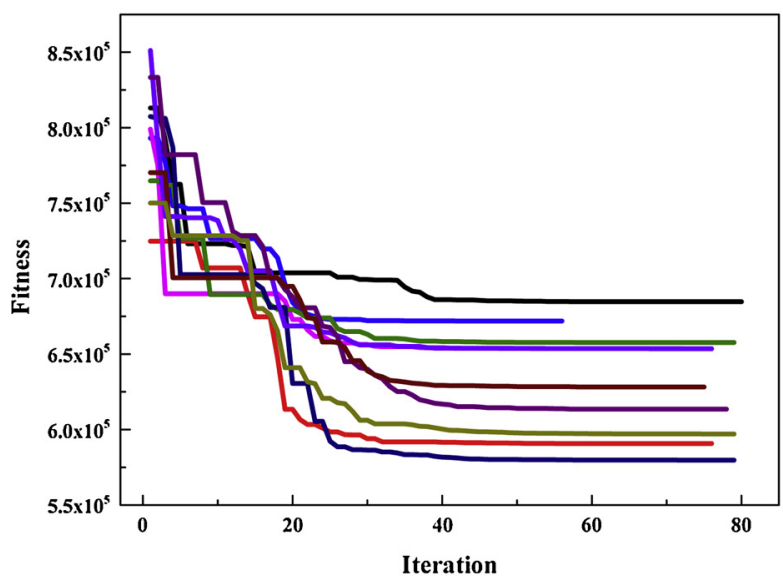

( c) SIPSO

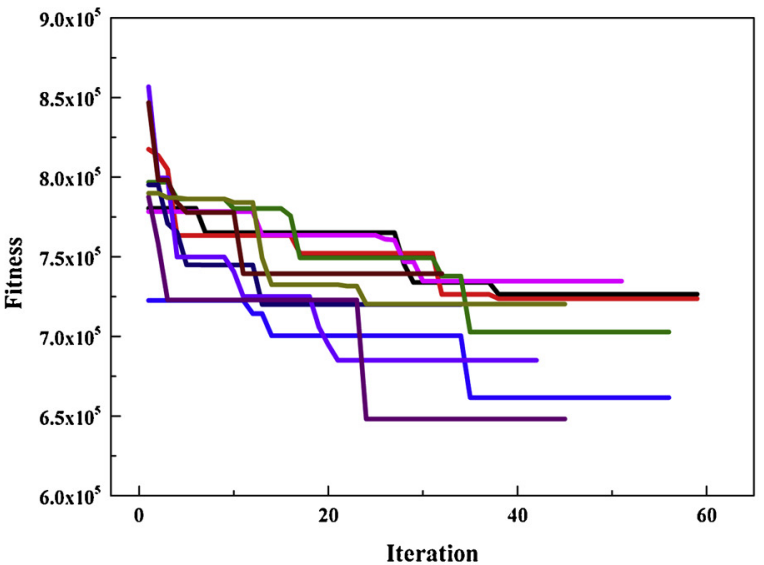

( b ) LPSO

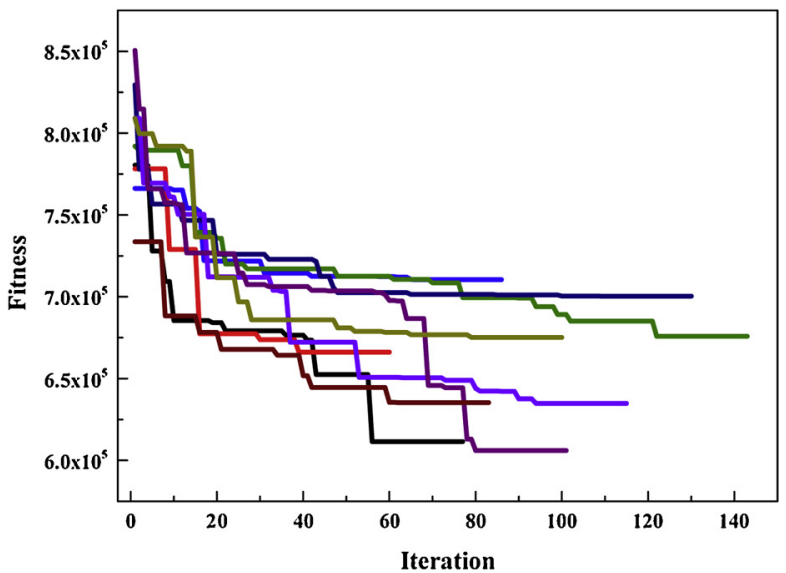

( d) MCPSO

Fig. 9. Results of four improved PSO algorithms.

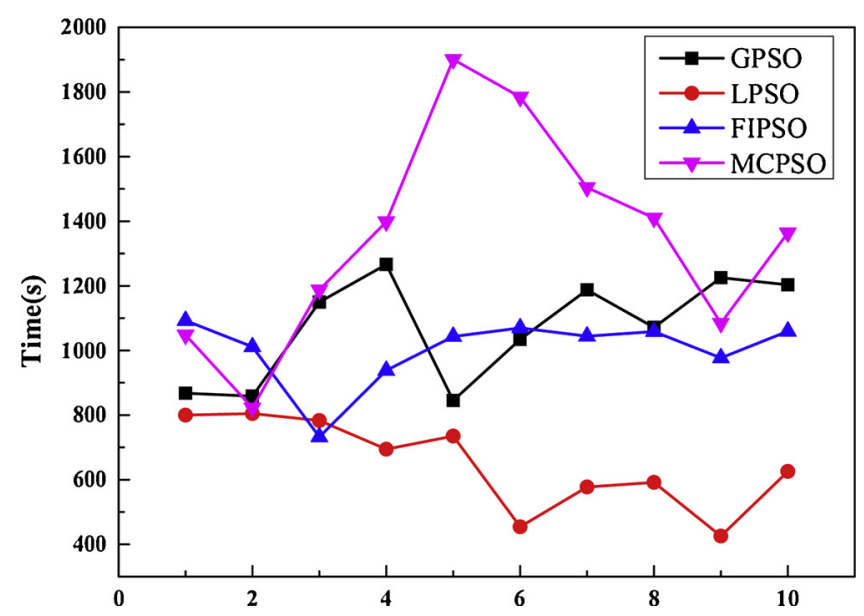

Fig. 10. Required time of four improved PSO algorithms.

$B_{i, k, j}^{S M N} \leq \frac{\sum_{r p \in R P} B_{i, k, r p}^{S M}+\sum_{r c \in R C} B_{k, j, r c}^{S N}}{2} \quad i \in I, \quad k \in K, j \in J$

$B_{i, k, j}^{S M N} \geq \frac{\sum_{r p \in R P} B_{i, k, r p}^{S M}+\sum_{r c \in R C} B_{k, j, r c}^{S N}-1}{2} \quad i \in I, k \in K, j \in J$ $\sum_{r \in R} B_{k, r}^{W} \leq 1 \quad k \in K$

$\sum_{r p \in R P} B_{i, k, r p}^{S M} \leq 1 \quad i \in I, \quad k \in K$

$\sum_{r c \in R C} B_{k, j, r c}^{S N} \leq 1 \quad k \in K, j \in J$

\subsubsection{Median distance}

The reason for the difficulty in convergence of BLD algorithm is the adoption of shortest distance, $D_{i, r}^{L}$ and $D_{j, r}^{L}$, which are shown in (6a) and (6b). Usually, the area of blocks is still large after the multiple division which leads to the big gap and difficulty in achieving convergence. Hence, the median distance, $D_{j, r}^{C}$ and $D_{i, r}^{L}$, are adopted instead of the shortest distance. Just as shown in Fig. 7 , the red line $\mathrm{AB}$ will be replaced by the golden line AC. When adopting the shortest distance, the maximum distance difference between $\mathrm{H} 1$ and $\mathrm{T} 3$, as part of gap, is represented by line $\mathrm{BD}$. In contrast, the maximum distance difference between $\mathrm{H} 1$ and $\mathrm{T} 3$ is represented by line $\mathrm{CF}$ when adopting the median distance.

$f 3_{k, j, r}=\alpha_{j} B_{k, j, r}^{S N} D_{j, r}^{C} \quad k \in K, \quad j \in J, \quad r \in R$

$f 4_{i, k, r}=B_{i, k, r}^{S M} D_{i, r}^{C} \quad i \in I, \quad k \in K, \quad r \in R$ 


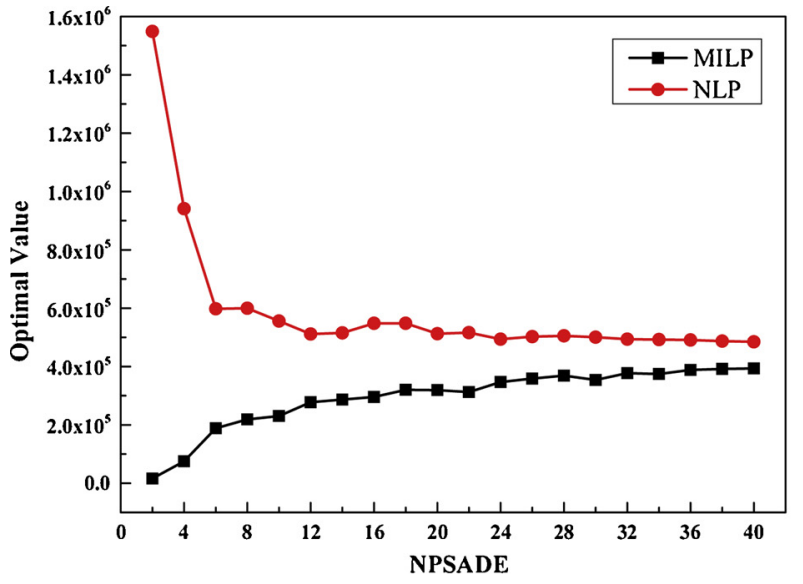

(a) Convergence process

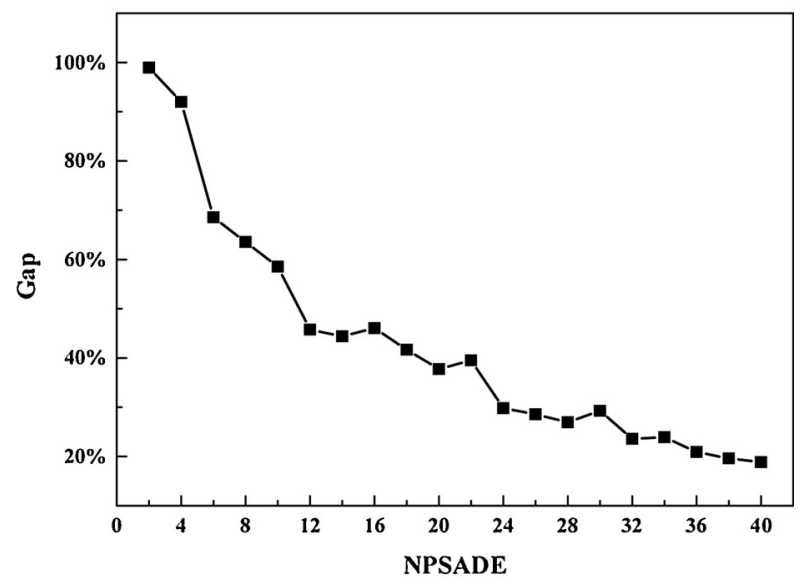

( b ) Gap

Fig. 11. Results of BLD algorithm.

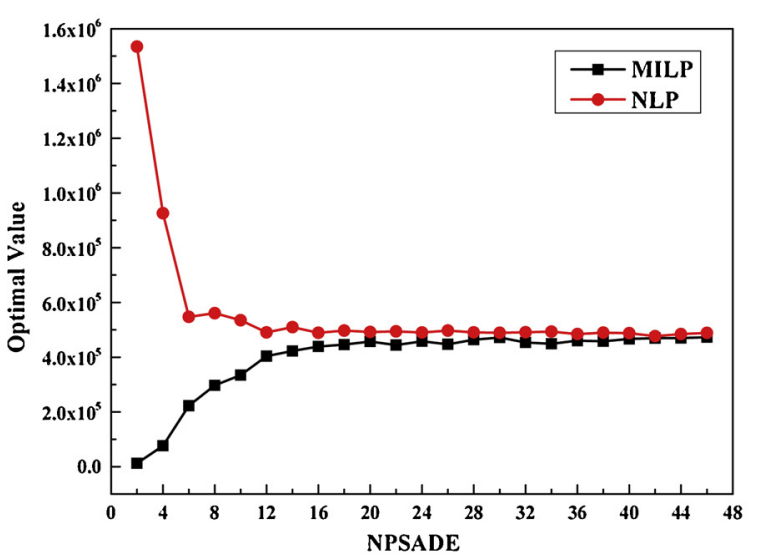

(a) Convergence process

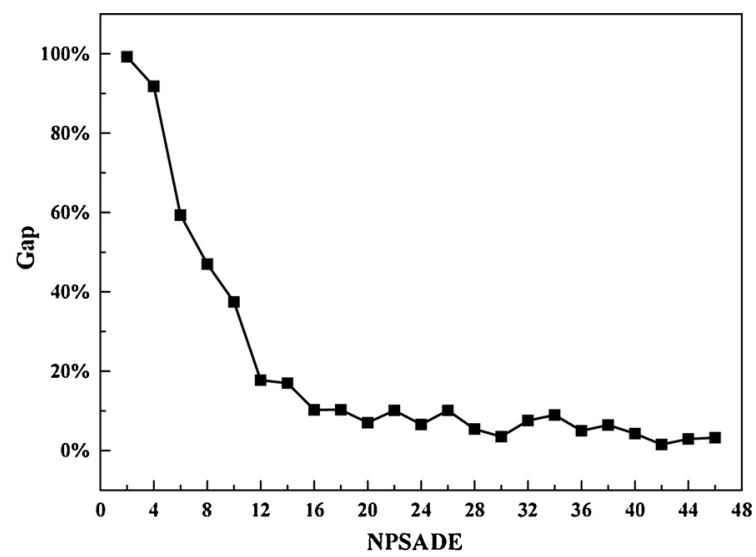

(b) Gap

Fig. 12. Results of ABLD algorithm for the 1st stage.

In this way, the possible difference between MILP model and NLP model is greatly reduced and then, gap will be greatly reduced as well. Especially, considering the solution of MILP model may be larger than or less than that of NLP model, the convergence condition for gap is changed from reaching the pre-specified optimality tolerance to reaching the pre-specified optimality tolerance multiple times.

\section{Results and discussion}

\subsection{Basic data}

As known information, we used dataset of hospitals and trapped people which are gathered from Homeland Infrastructure FoundationLevel Data (HIFLD) and tweet for hurricane Harvey in our previous work (Schempp et al., 2019). In the case, there are 15 hospitals and 94 places with tapped people in the disaster area, which is a square shape with a side length of $64,532 \mathrm{~m}$, as shown in Fig. 8. Since the area is a two-dimensional plane, the coordinates of hospitals and places with trapped people are represented by two arrays of $x$ and $y$. Each hospital has the certain capacity of providing service for the patients, reflected in the number of beds they own. The information is shown in Table 2. As the rescue proceeds, places with trapped people are found gradually. The rescue lasts for three days in the disaster and 28, 47 and 19 places are found in the first, second and third days respectively. In the problem, each day will be treated as a stage. After data collection and organization, the number of trapped people and degree of urgency of each place can be obtained. The data is important to the development of rescue plan. The information of each place is shown in tables in appendix because of the large amount.

In addition, as basic data of the established mathematical model, these parameters are shown in Table 3 . The parameters include the speed of rescue vehicles, the time needed to set up a rescue center at a possible location, pre-specified optimality tolerance, service scope of hospitals and rescue centers.

\subsection{Case study}

Matlab R2015a with Gurobi solver on a computer with Intel(R) Xeon(R) CPU E5-2640 v4 @ 2.40 GHz and 64GB of RAM is employed to solve the problem. The results are shown as follows.

\subsubsection{Comparison of algorithms on the first stage}

In this paper, three algorithms are adopted to solve the established MINLP model to obtain the optimal rescue plan in the disaster. And the comparison among the three algorithms will be based on three aspects which are related to the required time, stability and quality of the 


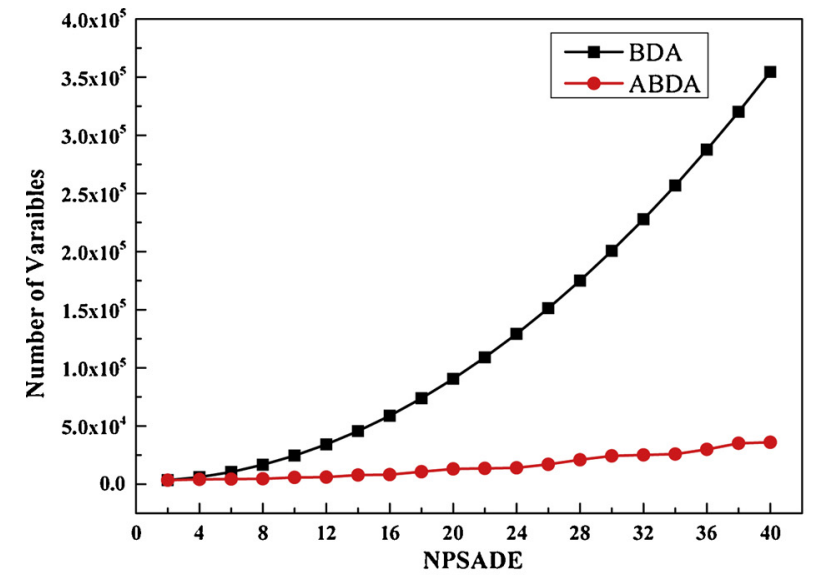

( a ) Number of variables

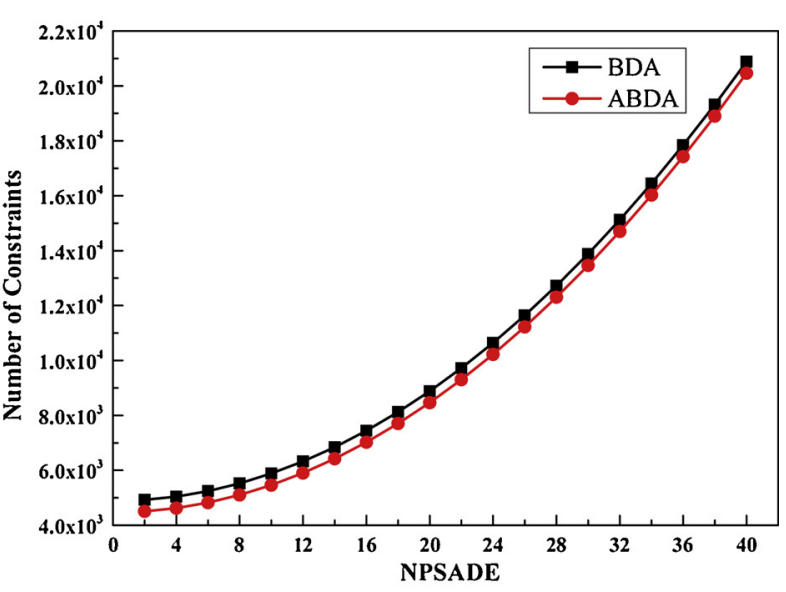

(b ) Number of constraints

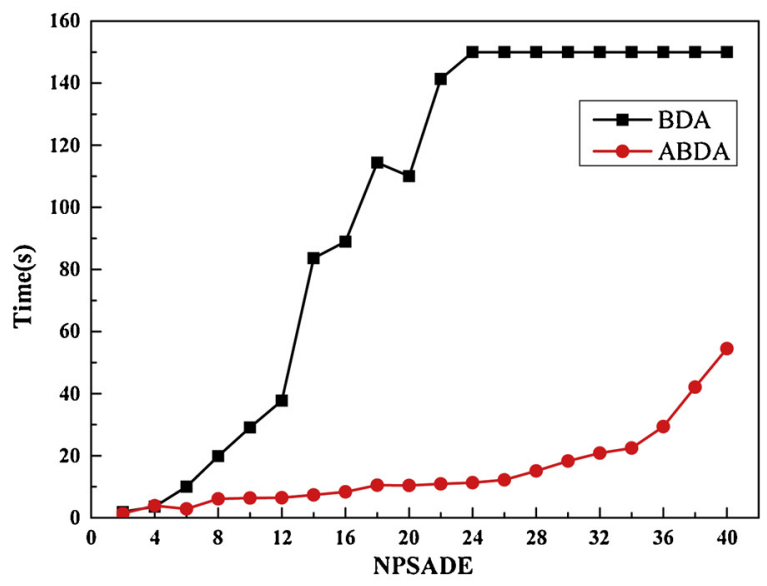

(c) Required time

Fig. 13. Comparison between BLD algorithm and ABLD algorithm.

Table 4

Coordinates of rescue centers for the 1st stage.

\begin{tabular}{llllll}
\hline Rescue center & $\mathrm{X} / \mathrm{m}$ & $\mathrm{Y} / \mathrm{m}$ & Rescue center & $\mathrm{X} / \mathrm{m}$ & $\mathrm{Y} / \mathrm{m}$ \\
\hline R1 & 54,673 & 17,756 & $\mathrm{R} 3$ & 53,971 & 13,826 \\
R2 & 26,746 & 12,697 & R4 & 55,296 & 29,184 \\
\hline
\end{tabular}

results. Here, the first stage is taken as an example.

6.2.1.1. PSO algorithm. Four improved PSO algorithms are taken to carry out ten tests respectively, including GPSO, LPSO, SIPSO and MCPSO. The results are shown in Figs. 9 and 10. Solution stability and results can be obtained from Fig. 9, where the ordinate represents the optimal value of the model and the abscissa represents the number of iterations during each test. Firstly, as Fig. 9 shown, the convergence of PSO algorithms in solving the problem is poor because the four PSO algorithms do not converge to the same value during ten tests respectively. Especially for SIPSO, the results of ten tests are the most scattered. Secondly, the best results of the four algorithms are 573,897 , $648,156,579,793,605,997$. Therefore, GPSO is more likely to obtain a good solution. Moreover, the required time of the four algorithms is shown in Fig. 10. Usually, LPSO takes less time while MCPSO takes longer because MCPSO can jump out of the local optimal solution in the process of solving while LPSO cannot. LPSO and SIPSO take almost the same amount of time. Through the above comparison, GPSO performs best in the four improved algorithms.

6.2.1.2. Bilevel decomposition algorithm. During the process of solving by BLD algorithm, the disaster area will be divided into more blocks after iterations. When the number of parts that the length of the area is divided equally into (NPLADE) reached 40, the solution is terminated due to out of memory.

Results are shown in Fig. 11. It can be seen that the optimal value of MILP and NLP models get closer as the number of blocks increases gradually from the figure (a). In the figure, the ordinate represents NPLADE and the abscissa represents the optimal value. However, when NPLADE is greater than 10 , the speed of the optimal value getting closer has obviously decreased. It means that by BLD algorithm, the convergence speed will be greatly reduced when the area is divided into a certain number of blocks, which is shown in figure (b). The slope of the curve tends to decrease gradually with the increase of NPLADE. The final gap is $18.9 \%$, which does not meet the converge requirement $\varepsilon=10 \%$.

In spite of this, BLD algorithm performs better than PSO algorithms 


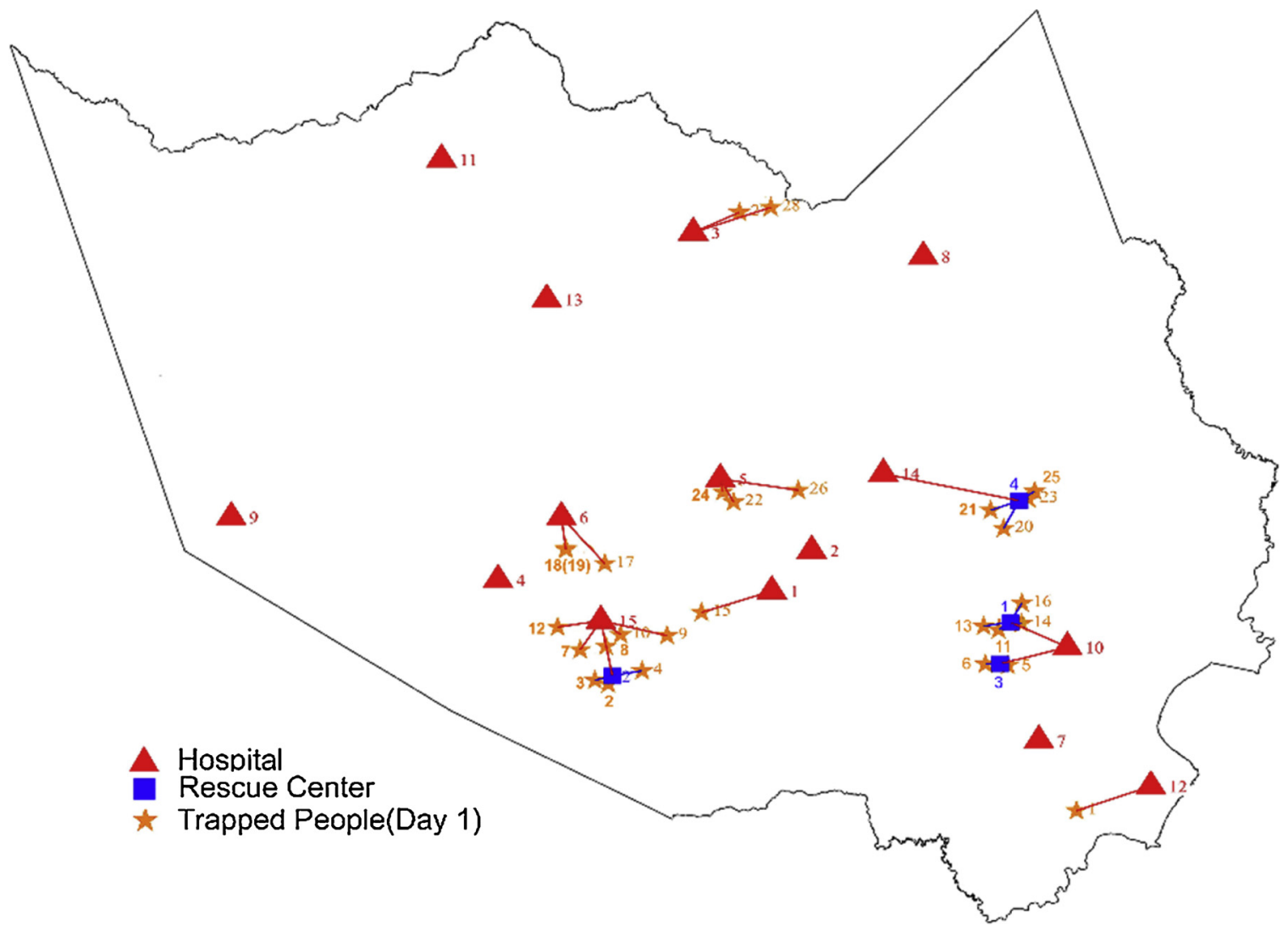

Fig. 14. Connections for the 1st stage.

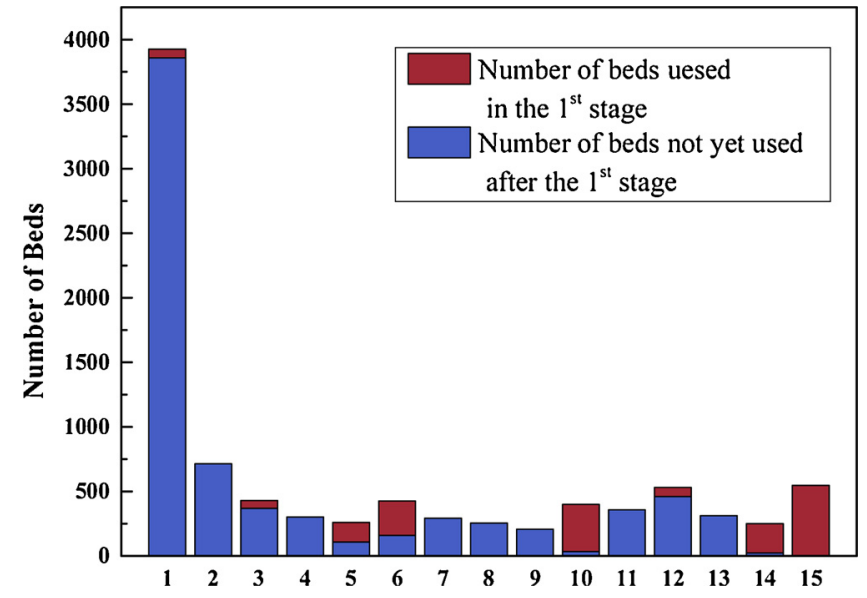

Fig. 15. Beds of hospitals for the 1st stage.

in three aspects. Compared with the instability of PSO algorithms, BLD algorithm is always convergent and a certain result can be obtained. When it comes to the quality of the result, the optimal value of BLD algorithm is 485,025 , which is better than 573,897 , the best result of PSO algorithms. In addition, the total required time of BLD algorithm is $1991 \mathrm{~s}$ while the average required a time of GPSO is $1071 \mathrm{~s}$ during the ten tests. And the required time of BLD algorithm can be greatly reduced if the step length of NPLADE is increased from 2 to 4 or 6 .

6.2.1.3. Accelerated bilevel decomposition algorithm. Here, ABLD algorithm is taken to solve the established model and the results are shown in Fig. 12. When NPLADE reaches 46, the solution is terminated because the convergence requirement has been met, reflected in that gap has been less than $\varepsilon$ for ten times in a row. And the optimal value of
ABLD algorithm is 476,593 . Combining figure (a) and figure (b), it can be seen that ABLD algorithm converges very quickly in solving the problem due to the adoption of median distance. The optimal value of MILP and NLP model get quite close when NPLADE reaches 12 and when NPLADE reaches 20, gap meets the requirement for the first time. Since NPLADE is larger than 26, gap had been less than $\varepsilon$ until the solution is terminated.

From figure (c) in Fig. 13, it's obvious that the required time of ABLD algorithm is far less than that of BLD algorithm. The process of NPLADE less than 40 is taken for comparison considering that BLD algorithm is terminated when NPLADE reaches 40 . The total required time of ABLD algorithm is $301 \mathrm{~s}$ while that of BLD algorithm is $1991 \mathrm{~s}$. The reason for that is the model scale of MILP is greatly reduced when taking ABLD algorithm instead of BLD algorithm, which is shown in figure (a) and figure (b) in Fig. 13. Due to the idea of establishing a model of the two algorithms is the same, a number of constraints is similar. However, a number of variables is completely different because service scope is adopted in ABLD algorithm. It can be seen in figure (a) that the number of variables using ABLD algorithm is far less than that using BLD algorithm, especially when NPLADE is large. In addition to this, when it comes to the quality of results of the two algorithms, ABLD algorithm is still better than BLD algorithm with 476,593 less than 485,025 . And the reason for that is ABLD algorithm has converged before terminated while BLD algorithm has not. Therefore, ABLD algorithm will be taken to solve the second and third stage of the problem because more places with trapped people will be found, which leads to great difficulty in solving.

Here are the results of the first stage, including connections among nodes, coordinates of rescue centers which has been set up and the condition of usage of beds in hospitals. In the first stage, four rescue centers are set up just as shown in Table 4 and the layout is shown in Fig. 14. From Fig. 14, it can be seen that rescue centers are usually set up at the locations which are close to many places with trapped people, 


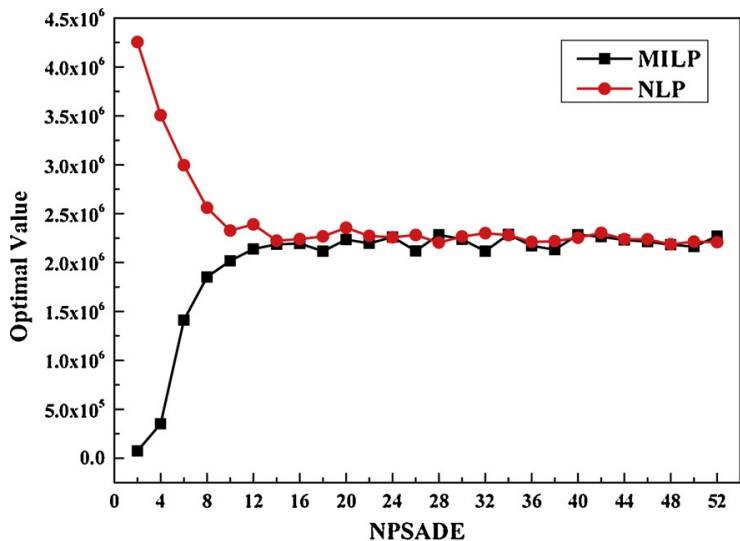

(a) Convergence process

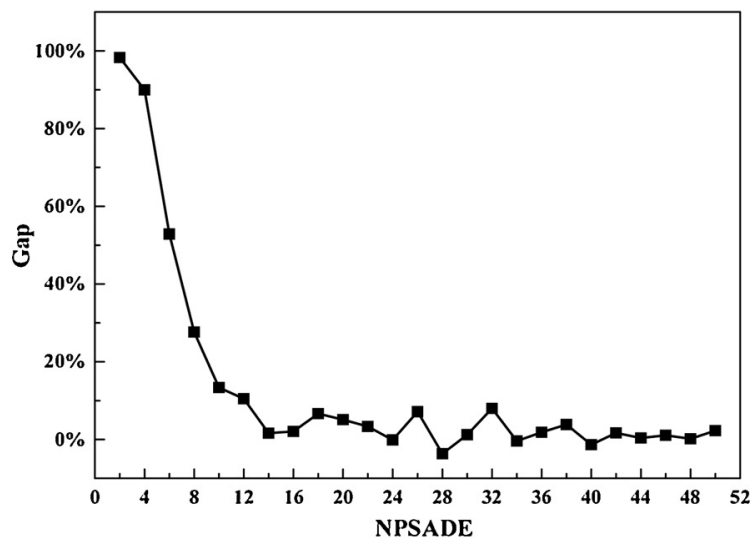

(b) Gap

Fig. 16. Results of ABLD algorithm for the 2nd stage.

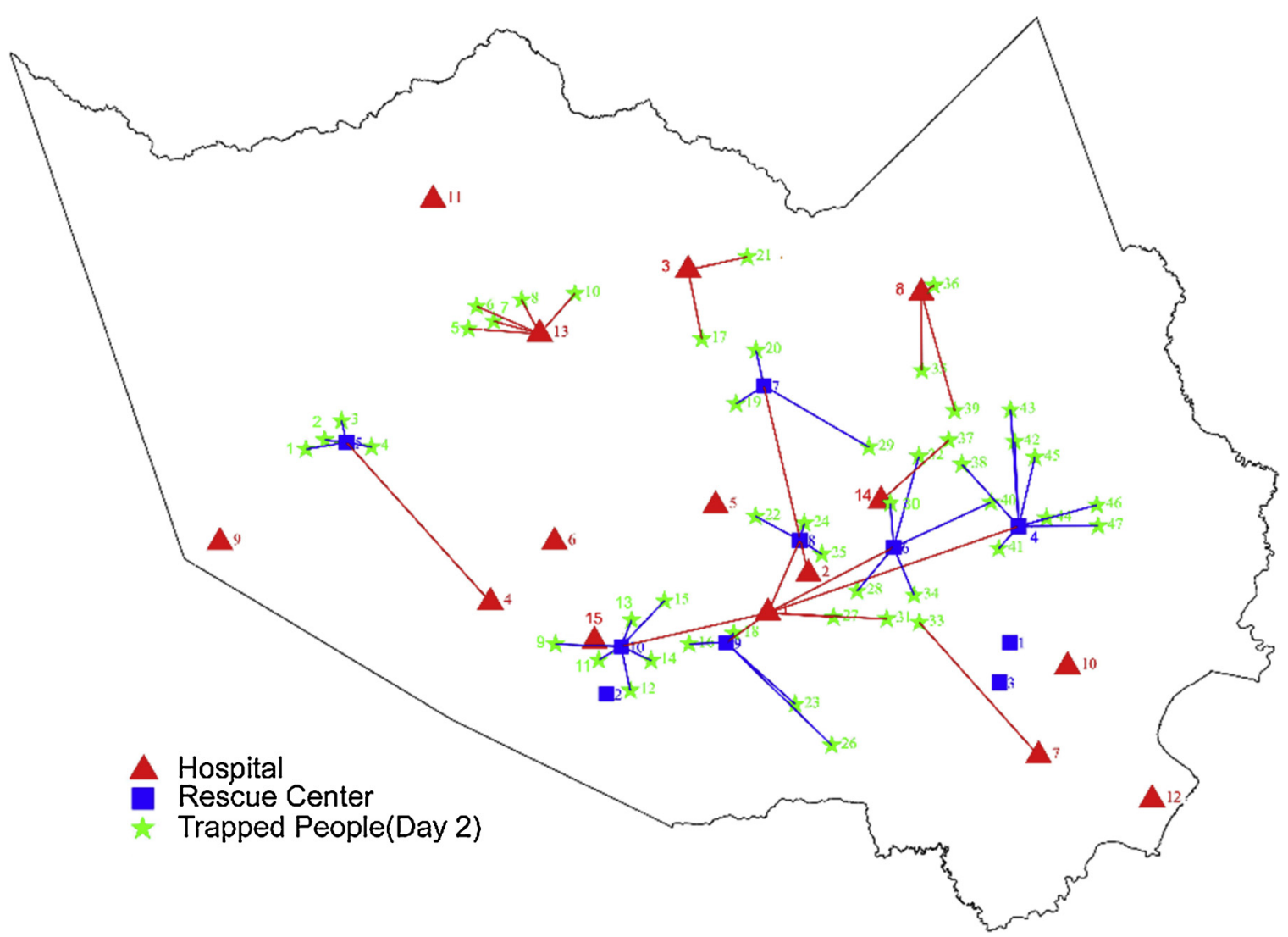

Fig. 17. Connections for the 2nd stage.

especially R1 and R4. Taking rescue centers as transfer stations, the total distance to send people to the hospital will be greatly reduced. To the people located in places which are close to hospitals or isolated from other places, they are often sent to hospitals directly, as T22 and T24 do. The condition of usage of beds in hospitals can be seen in Fig. 15. In this stage, all the beds in $\mathrm{H} 15$ are used, which means that no people could be sent to H15 in the next stages. Similarly, most of the beds in $\mathrm{H} 10$ and $\mathrm{H} 14$ has been used, which leads to that they could receive a few people in the next stages.

\subsubsection{Results of the second stage}

In the second stage, when solving the problem, four rescue centers set up in the first stage should be taken into consideration. The convergence process and curve of gap are shown in Fig. 16. Although there are 47 places with trapped people found in this stage which leads to the large model scale, it still converges fast by ABLD algorithm. From figures (a) and (b), the optimal value of MILP and NLP models get close when NPLADE reaches 14 . However, the convergence in the early is unstable due to the large model scale, reflected in that gap has large fluctuation when NPLADE is less than 32. Finally, when NPLADE reaches 50 , the solution is terminated.

The results of the second stage are shown in Figs. 17 and 18 and Table 5. Another six rescue centers have been set up in this stage. And the new rescue centers are all set up at the locations which are close to 


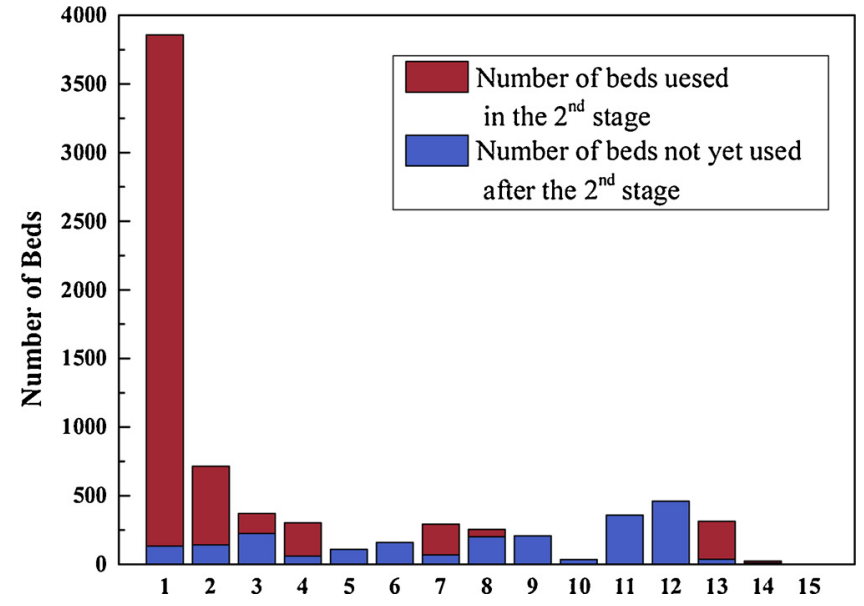

Fig. 18. Beds of hospitals for the 2nd stage.

Table 5

Coordinates of rescue centers for the 2nd stage.

\begin{tabular}{llllll}
\hline Rescue center & X/m & Y/m & Rescue center & X/m & Y/m \\
\hline R5 & 8758 & 37,449 & R8 & 40,136 & 27,821 \\
R6 & 46,629 & 27,122 & R9 & 35,035 & 17,735 \\
R7 & 37,651 & 43,020 & R10 & 27,770 & 17,366 \\
\hline
\end{tabular}

many places with trapped people, for example, R5 and R10, which is similar to the first stage or even more obvious because the number of places found increases a lot. To the people located in places which are close to hospitals or isolated from other places, they are still sent to hospitals directly, such as H5-H10. In addition, there are three points worth attention. Due to the large number of beds which are not yet used in $\mathrm{H} 1$, a large number of people are still sent to $\mathrm{H} 1$ although it's not at the center of the disaster area. As a rescue center set up in the previous stage, R4 is connected to many places although it's not at the center of the locations of the places, which can save the time to set up a new rescue center. Because there are no places with trapped people close to $\mathrm{R} 1$ and R3, they are not used in this stage.

\subsubsection{Results of the third stage}

Similar to the second stage, the ten rescue centers which has been set up need to be considered in the third stage. From Fig. 19, the solution is terminated when NPLADE reaches 38 . It converges fast and the convergence process is stable due to the smaller model scale compared

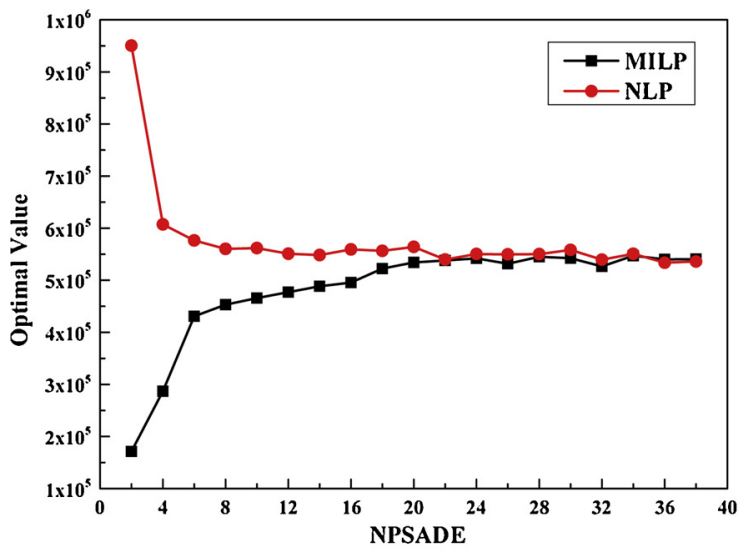

( a ) Convergence process with the previous stages with 19 places found in this stage.

The results of the third stage are shown in Figs. 20 and 21 and Table 6. Two new rescue centers are set up in this stage, R11 connected to T4 and T7 and R12 connected T12, T14 and T18. Moreover, R1, R4, $\mathrm{R} 5, \mathrm{R} 6$ and R7 which has been set up in the previous stage are used, for example, T15 and T17 are connected to H9 with the transfer station R5. In this stage, many trapped people have to be sent to the hospitals farther away because most beds in many hospitals have been used. For example, T9, T11 and T16 are connected to H2 by taking R6 as the transfer station due to the little number of beds in H14. Especially, R4 is connected to $\mathrm{H} 11$ because there are few beds left in the hospitals around it. And here, the service scope of $\mathrm{H} 11$ is increased from $20,000 \mathrm{~m}$ to $64,532 \mathrm{~m}$, which is the side length of the disaster area.

\section{Conclusion}

Instead of developing a rescue plan by human expertise manual decision-making based on previous rescue experience, an MINLP model is established to obtain a rescue plan with the highest rescue efficiency in this paper. To solve the model, the PSO algorithm and BLD algorithm are adopted. The two algorithms both convert the MINLP model into the MILP model whose solution method has been comprehensive. Moreover, considering the large model scale in the problem, accelerated solutions are proposed for generating BLD algorithm and ABLD algorithm. Then a real-world disaster rescue is taken as a case study, which is composed of three stages. Each stage should be based on the previous stage except the first stage because some rescue centers have been set up. The comparison among four improved PSO algorithms, BLD algorithm and ABLD algorithm is conducted when solving the problem of the first stage. GPSO performs best in the four improved algorithms because its best solution is the 573,897 , which is minimal and its required time and stability are relatively superior. BLD algorithm outperforms GPSO, reflected in that the stability is very high and the quality of results is better, whose optimal value are 485,025 and 573,897 separately. However, the convergence requirement is not met and the required time is relatively long when using BLD algorithm. The problems do not exist when using ABLD algorithm because when NPLADE reaches 46 , the convergence requirement has been met and the total required time of ABLD algorithm is $301 \mathrm{~s}$ while that of BLD algorithm is $1991 \mathrm{~s}$. In addition, the optimal value of ABLD algorithm is 476,593 which is better than that of BLD algorithm. Hence, the second and the third stage of the problem are solved by ABLD algorithm to obtain the optimal rescue plans.

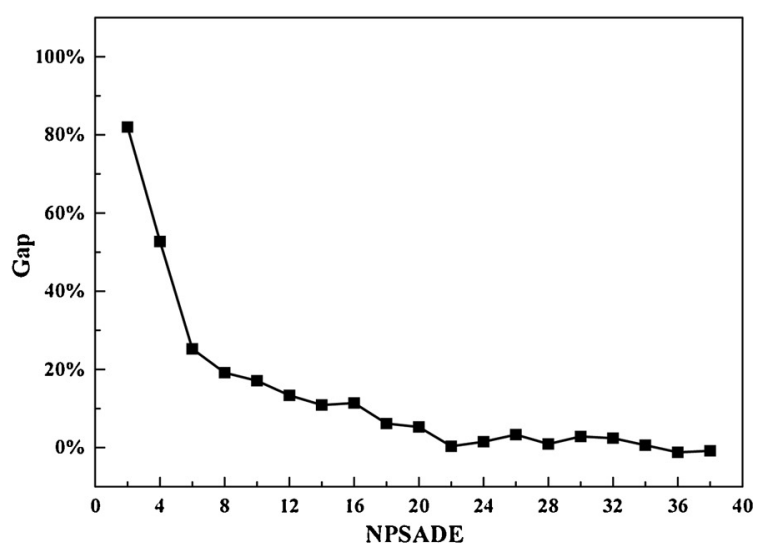

Fig. 19. Results of ABLD algorithm for the 3rd stage. 


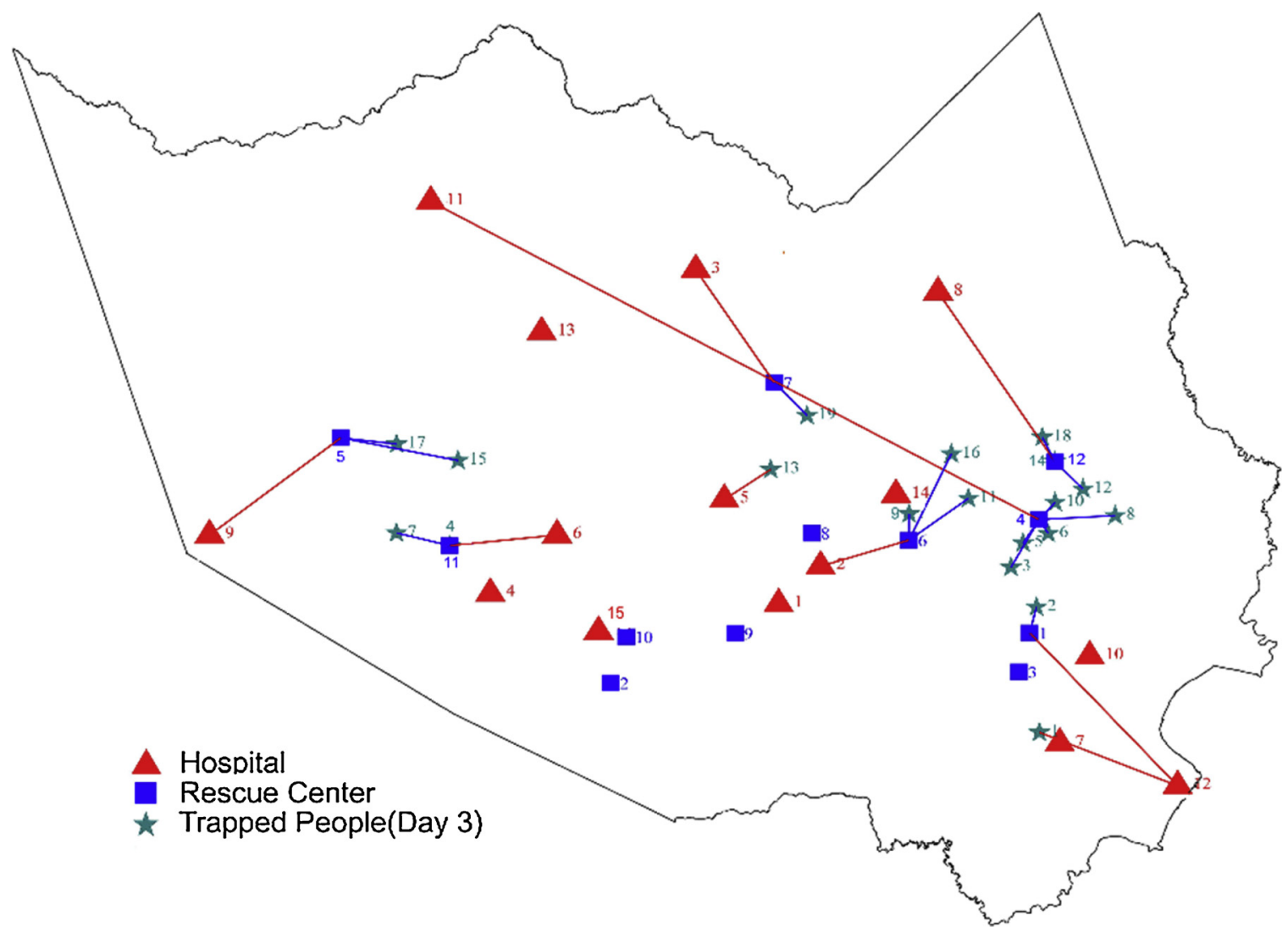

Fig. 20. Connections for the 3rd stage.

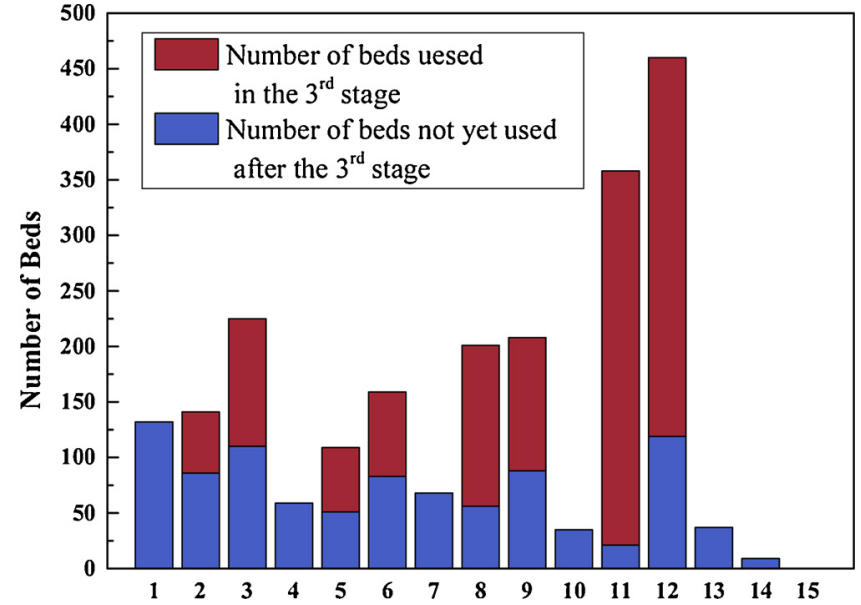

Fig. 21. Beds of hospitals for the 3rd stage.

Table 6

Coordinates of rescue centers for the 3rd stage.

\begin{tabular}{llllll}
\hline Rescue center & $\mathrm{X} / \mathrm{m}$ & $\mathrm{Y} / \mathrm{m}$ & Rescue center & $\mathrm{X} / \mathrm{m}$ & $\mathrm{Y} / \mathrm{m}$ \\
$\mathrm{R} 11$ & 16,024 & 26,564 & $\mathrm{R} 12$ & 56,344 & 35,076 \\
\hline
\end{tabular}

\section{Nomenclature}

Sets and indicesi $\in I=\left\{1,2, \ldots, i_{\max }\right\}$ set of all hospitals in the rescue system. $i_{\max }$ represents the maximum number of hospitalsk $\in K=\left\{1,2, \cdots, k_{\max }\right\}$ set of all the locations in the rescue system. $k_{\max }$ represents the maximum number of locationsk $1 \in K 1=\left\{1,2, \ldots, k 1_{\max }\right\}$ set of the locations where rescue centers have been set up in the previous stage. $k 1_{\max }$ represents the maximum number of locations $k \in K 2=\left\{1,2, \ldots, k 2_{\max }\right\}$ set of all the locations where rescue centers are set up in the non-first stage. $k 2_{\max }$ represents the maximum number of locations $j \in J=\left\{1,2, \ldots, j_{\max }\right\}$ set of all trapped persons in the rescue system. $j_{\max }$ represents the maximum number of trapped persons $r \in R=\left\{1,2, \ldots, r_{\max }\right\}$ set of all blocks divided in the rescue system. $r_{\max }$ represents the maximum number of blocksrc $\in R C=\left\{1,2, \cdots, r c_{\max }\right\}$ set of blocks that could be served by each rescue center. $r c_{\max }$ represents the maximum number of the blocksrp $\in R P=\left\{1,2, \ldots, r p_{\max }\right\}$ set of blocks that could be served by each hospital. $r p_{\max }$ represents the maximum number of the blocksContinuous parameters $C_{i}^{A}$ the number of patients that could be received by the hospital $i D_{i, r}^{L}, D_{i, r p}^{L}, D_{j, r}^{L}, D_{j, r c}^{L}$ the shortest distance between hospital $i$ and block $r, \operatorname{km} D_{i, r}^{C}, D_{i, r p}^{C}, D_{j, r}^{C}, D_{j, r c}^{C}$ the distance between hospital $i$ and the center of block $r, \mathrm{~km} M$ the sufficiently large num$\operatorname{ber} P_{j}^{E}$ the number of trapped people found at the location $j P_{k}^{U}$ the time needed to set up a rescue center at possible location $k, \min S^{H}$ service scope of hospitals and rescue centers, $\mathrm{km} S^{R C}$ service scope of rescue centers, $\mathrm{km} V$ speed of rescue vehicles, $\mathrm{km} \mathrm{h}^{-1} x_{\text {down }}, x_{\text {up }}, y_{\text {down }}, y_{u p}$ the upper and lower limits of the coordinates of the block where rescue center $k$ is located, $\mathrm{km}_{j}$ the degree of urgency to rescue the trapped people jgapthe difference between the lower and upper bound in BLD algorithm and ABLD algorithmea pre-specified optimality tolerance in BLD algorithm and ABLD algorithmPositive continuous variables $f 1_{k}$, $f 1_{k, r}$ the corresponding distance to the time needed to set up the rescue centers, $\operatorname{km} f 2_{i, j}$ the distance needed to transport trapped person $j$ to hospital $i, \operatorname{kmf} 3_{k, j, r}, f 3_{k, j, r}$ the distance needed to transport trapped person $j$ to possible location of rescue center $k$ which is located in block $r$ or $r c$ and trapped person $j$ is within the service scope of rescue center $k, \operatorname{km} f 4_{i, k, r}, f 4_{i, k, r p}$ the distance needed to travel from possible location of rescue center $k$ which is located in block $r p$ to hospital $i$ and block $r$ or $r p$ is within the service scope of hospital $i, \operatorname{km} D_{i, j}, D_{i, k}, D_{k, j}$ the distance between two nodes, i.e., $D_{i, j}$ represents the distance between hospital $i$ and trapped person $j, \operatorname{km} x_{k} y_{k}$ the coordinates of possible 
location of rescue center $k$, kmBinary variables $B_{k}^{W}$ if a rescue center is set up at possible location $k, B_{k}^{W}$ equals 1 ; otherwise, $B_{k}^{W}$ equals $0 B_{k, r}^{W}$ if a rescue center is set up at possible location $k$ which is located in block $r$, $B_{k, r}^{W}$ equals 1 ; otherwise, $B_{k, r}^{W}$ equals $0 B_{i, k}^{S M}, B_{k, j}^{S N}$ if injured people are transported from rescue center $k$ to hospital $i, B_{i, k}^{S M}$ equals 1 ; otherwise, $B_{i, k}^{S M}$ equals $0 B_{i, k, r}^{S M}, B_{i, k, r p}^{S M}, B_{k, j, r}^{S N}, B_{k, j, r c}^{S N}$ if injured people are transported from rescue center $k$ which is located in block $r$ to hospital $i, B_{i, k, r}^{S M}$ equals 1 ; otherwise, $B_{i, k, r}^{S M}$ equals $0 B_{i, k, j}^{S M N}$ if trapped person $j$ are transported to hospital $i$ via rescue center $k, B_{i, k, j}^{S M N}$ equals 1 ; otherwise, $B_{i, k, j}^{S M N}$ equals 0

\section{Acknowledgement}

This work was partially supported by the National Natural Science Foundation of China (51874325), the Grant-in-Aid for Early-Career Scientists (19K15260) from the Japan Ministry of Education, Culture, Sports, Science and Technology, the Research Council of Norway (RCN), and the Norwegian Centre for International Cooperation in Education (SiU) grant through INTPART program.

\section{References}

Abounacer, R., Rekik, M., \& Renaud, J. (2014). An exact solution approach for multiobjective location-transportation problem for disaster response. Computers \& Operations Research, 41, 83-93.

Afshar, A., \& Haghani, A. (2012). Modeling integrated supply chain logistics in real-time large-scale disaster relief operations. Socio-Economic Planning Sciences, 46(4), $327-338$.

Altay, N., \& Green, W. G. (2006). OR/MS research in disaster operations management. European Journal of Operational Research, 175(1), 475-493.

Amin Hosseini, S. M., de la Fuente, A., \& Pons, O. (2016). Multi-criteria decision-making method for assessing the sustainability of post-disaster temporary housing units technologies: A case study in Bam, 2003. Sustainable Cities and Society, 20, 38-51.

Aras, N., Altınel, İ. K., \& Orbay, M. (2007). New heuristic methods for the capacitated multi-facility Weber problem. Naval Research Logistics (NRL), 54(1), 21-32.

Berkoune, D., Renaud, J., Rekik, M., \& Ruiz, A. (2012). Transportation in disaster response operations. Socio-Economic Planning Sciences, 46(1), 23-32.

Bozorgi-Amiri, A., Jabalameli, M. S., \& Al-E-Hashem, S. M. J. M. (2013). A multi-objective robust stochastic programming model for disaster relief logistics under uncertainty. OR Spectrum, 35(4), 905-933.

Cavdur, F., Kose-Kucuk, M., \& Sebatli, A. (2016). Allocation of temporary disaster response facilities under demand uncertainty: An earthquake case study. International Journal of Disaster Risk Reduction, 19, 159-166.

Chen, Y. H., Bai, J., \& Xing-Yuan, L. I. (2017). Model and algorithm for location-routing of multi-stage post-disaster emergency rescue. Industrial Engineering \& Management.

Choi, M., Starbuck, R., Lee, S., Hwang, S., Lee, S. H., Park, M., et al. (2018). Distributed and interoperable simulation for comprehensive disaster response management in facilities. Automation in Construction, 93, 12-21.

Chou, J.-S., Hsu, S.-C., Lin, C.-W., Lee, C.-J., \& Wei, J.-L. (2017). Structural analysis of the relationships between implementing tasks and ex-post effectiveness for disaster mitigation practice in Taipei City. Sustainable Cities and Society, 34, 1-11.

Das, R. (2018). Disaster preparedness for better response: Logistics perspectives. International Journal of Disaster Risk Reduction, 31, 153-159.

Doan, X. V., \& Shaw, D. (2019). Resource allocation when planning for simultaneous disasters. European Journal of Operational Research, 274(2), 687-709.

Galindo, G., \& Batta, R. (2013). Review of recent developments in OR/MS research in disaster operations management. European Journal of Operational Research, 230(2), 201-211.

Hayes, P., \& Kelly, S. (2018). Distributed morality, privacy, and social media in natural disaster response. Technology in Society, 54, 155-167.

Hu, Z. H., \& Sheng, Z. H. (2015). Disaster spread simulation and rescue time optimization in a resource network. Information Sciences, 298(C), 118-135.

Iqbal, S., Sardar, M. U., Lodhi, F. K., \& Hasan, O. (2018). Statistical model checking of relief supply location and distribution in natural disaster management. International Journal of Disaster Risk Reduction, 31, 1043-1053.

Jamali, M., Nejat, A., Ghosh, S., Jin, F., \& Cao, G. (2019). Social media data and postdisaster recovery. International Journal of Information Management, 44, 25-37.

Jian, T., Zhu, K., Guo, H., Gong, C., Liao, C., \& Zhang, S. (2018). Using auction-based task allocation scheme for simulation optimization of search and rescue in disaster relief. Simulation Modelling Practice \& Theory, 82, 132-146.

Kontokosta, C. E., \& Malik, A. (2018). The Resilience to Emergencies and Disasters Index: Applying big data to benchmark and validate neighborhood resilience capacity. Sustainable Cities and Society, 36, 272-285.

Lara, C. L., Trespalacios, F., \& Grossmann, I. E. (2018). Global optimization algorithm for capacitated multi-facility continuous location-allocation problems. Journal of Global Optimization, 71(4), 871-889.

Lei, Z., Wu, X., Xu, Z., \& Fujita, H. (2017). Emergency decision making for natural disasters: An overview. International Journal of Disaster Risk Reduction, 27, 567-576.

Miller, W. (2015). What does built environment research have to do with risk mitigation, resilience and disaster recovery? Sustainable Cities and Society, 19, 91-97.

Okubo, T. (2016). Traditional wisdom for disaster mitigation in history of Japanese Architectures and historic cities. Journal of Cultural Heritage, 20, 715-724.

Pagano, A., Pluchinotta, I., Giordano, R., \& Vurro, M. (2017). Drinking water supply in resilient cities: Notes from L'Aquila earthquake case study. Sustainable Cities and Society, 28, 435-449.

Pérez-Rodríguez, N., \& Holguín-Veras, J. (2016). Inventory-allocation distribution models for postdisaster humanitarian logistics with explicit consideration of deprivation costs. Transportation Science, 50(4), 1261-1285.

Rauchecker, G., \& Schryen, G. (2019). An exact branch-and-price algorithm for scheduling rescue units during disaster response. European Journal of Operational Research, 272(1), 352-363.

Royero, D. R., Pacheco, G. G., \& Pinedo, R. Y. (2016). A dynamic model for disaster response considering prioritized demand points. Socio-Economic Planning Sciences, 55, 59-75.

Sánchez-García, J., Reina, D. G., \& Toral, S. L. (2019). A distributed PSO-based exploration algorithm for a UAV network assisting a disaster scenario. Future Generation Computer Systems, 90, 129-148.

Schempp, T., Zhang, H., Schmidt, A., Hong, M., \& Akerkar, R. (2019). A framework to integrate social media and authoritative data for disaster relief detection and distribution optimization. International Journal of Disaster Risk Reduction, (9, April, 2019), 101143.

Sherali, H. D., Al-Loughani, I., \& Subramanian, S. (2002). Global optimization procedures for the capacitated Euclidean and $l_{p}$ distance multifacility location-allocation problems. Operations Research, 50(3), 433-448.

Sommerfeldt, E. J. (2015). Disasters and information source repertoires: Information seeking and information sufficiency in postearthquake Haiti. Journal of Applied Communication Research, 43(1), 1-22.

Sun, Q. Y., \& Li, X. Y. (2014). The fault-tolerant framework of large-scale disasters based on continuity management: A case of power network infrastructure. 2014 International Conference on Management Science \& Engineering 21th Annual Conference Proceedings.

Tzeng, G. H., Cheng, H. J., \& Huang, T. D. (2007). Multi-objective optimal planning for designing relief delivery systems. Transportation Research Part E, 43(6), 673-686.

Wex, F., Schryen, G., Feuerriegel, S., \& Neumann, D. (2014). Emergency response in natural disaster management: Allocation and scheduling of rescue units. European Journal of Operational Research, 235(3), 697-708.

Wohlgemuth, S. (2012). Dynamic vehicle routing with anticipation in disaster relief. Socio-Economic Planning Sciences, 46(4), 261-271.

Yan, L., Bao, J., Hu, X., \& Ye, J. (2009). A heuristic project scheduling approach for quick response to maritime disaster rescue. International Journal of Project Management, 27(6), 620-628.

Zhang, H., Song, X., Huang, D., Xu, N., Shibasaki, R., \& Liang, Y. (2019). EX-ante online risk assessment for building emergency evacuation through multimedia data. PLOS ONE, 14(4), e0215149.

Zhang, S., Guo, H., Zhu, K., Yu, S., \& Li, J. (2017). Multistage assignment optimization for emergency rescue teams in the disaster chain. Knowledge-Based Systems, 137, $123-137$. 